\title{
Evaluation of the Potential for Detection and Classification of Ailanthus altissima (Tree of Heaven) Using LiDAR Data
}

\author{
Cassidy Robert Rhea \\ West Virginia University
}

Follow this and additional works at: https://researchrepository.wvu.edu/etd

\section{Recommended Citation}

Rhea, Cassidy Robert, "Evaluation of the Potential for Detection and Classification of Ailanthus altissima (Tree of Heaven) Using LiDAR Data" (2012). Graduate Theses, Dissertations, and Problem Reports. 3521. https://researchrepository.wvu.edu/etd/3521

This Thesis is protected by copyright and/or related rights. It has been brought to you by the The Research Repository @ WVU with permission from the rights-holder(s). You are free to use this Thesis in any way that is permitted by the copyright and related rights legislation that applies to your use. For other uses you must obtain permission from the rights-holder(s) directly, unless additional rights are indicated by a Creative Commons license in the record and/ or on the work itself. This Thesis has been accepted for inclusion in WVU Graduate Theses, Dissertations, and Problem Reports collection by an authorized administrator of The Research Repository @ WVU. For more information, please contact researchrepository@mail.wvu.edu. 


\title{
Evaluation of the Potential for Detection and Classification of Ailanthus altissima (Tree of Heaven) Using LiDAR Data
}

\author{
Cassidy Robert Rhea \\ Thesis submitted to the \\ Eberly College of Arts and Sciences \\ At West Virginia University \\ in partial fulfillment of the requirements \\ For the degree of \\ Master of Arts \\ In \\ Geography \\ Committee Members: \\ Timothy Warner, Ph.D., Chair \\ Jamison Conley, Ph.D. \\ Rick Landenberger, Ph.D. \\ Department of Geology and Geography \\ Morgantown, West Virginia \\ 2012
}

Keywords: LiDAR, Intensity Values, Tree Species Classification, Ailanthus altissima 


\begin{abstract}
Evaluation of the Potential for Detection and Classification of Ailanthus altissima (Tree of Heaven) Using LiDAR Data
\end{abstract}

\author{
Cassidy R. Rhea
}

This thesis compares methods for delineating and classifying the invasive, exotic tree Ailanthus altissima (tree of heaven), using attributes derived entirely from light detection and ranging (LiDAR) data. The accuracy of two image segmentation methods: 1) Tree variable window program (TreeVaW) and 2) watershed segmentation, and three classifications schemes: 1) classification and regression trees (CART) 2) artificial neural networks (NN) and 3) support vector machines (SVM) are compared. I found that generally the watershed segmentation method produced better segmentation results than the TreeVaW segmentation method, and that the CART classification was the most accurate overall classifier, although the SVM classification produced the most accurate Ailanthus species classification. The factors that are most important in influencing the segmentation and classification accuracies are the point density of the LiDAR data, the level of tree-crown penetration by the LiDAR laser pulses, and the quality of the canopy height model derived from the LiDAR data point cloud. CART and SVM classification, together with watershed segmentation are optimal methods of identifying Ailanthus altissima trees from LiDAR data. 


\section{Table of Contents}

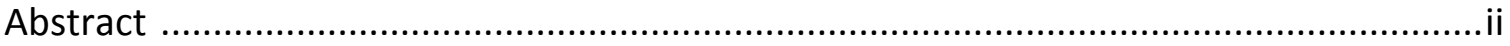

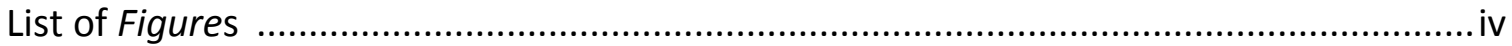

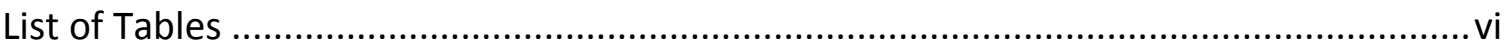

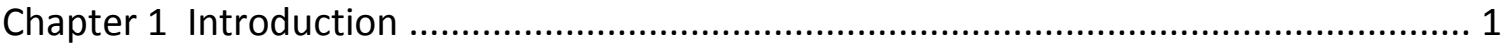

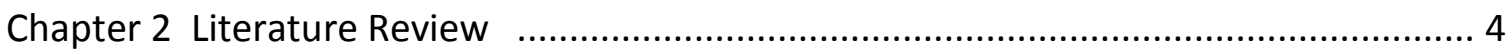

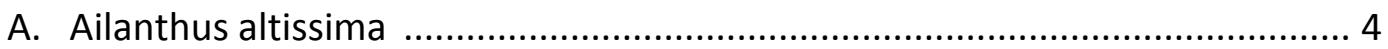

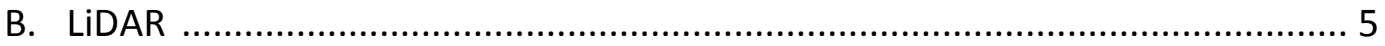

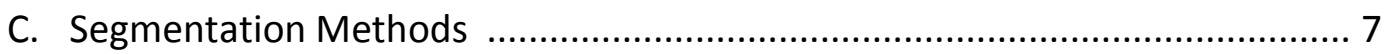

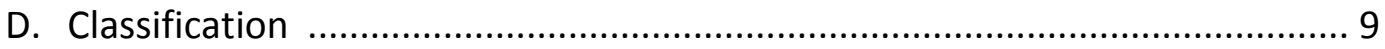

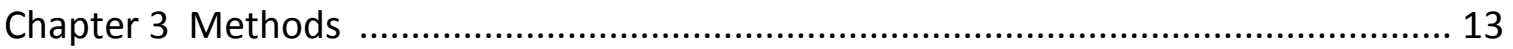

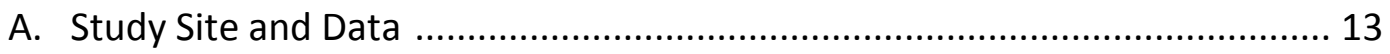

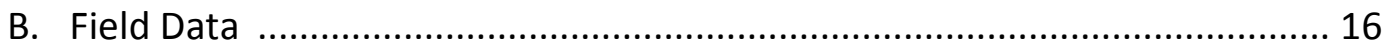

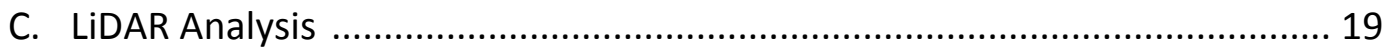

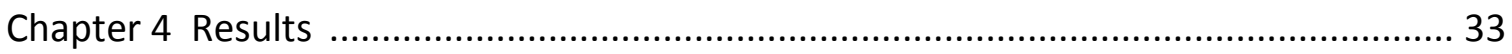

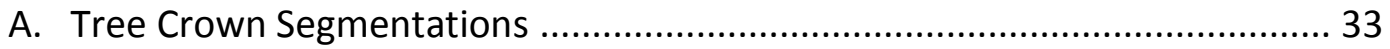

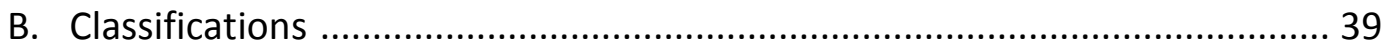

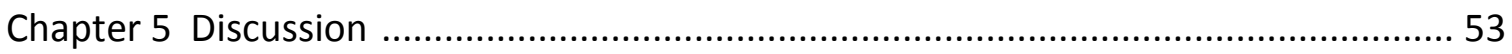

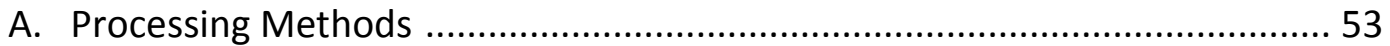

B. Segmentation Methods ........................................................................ 56

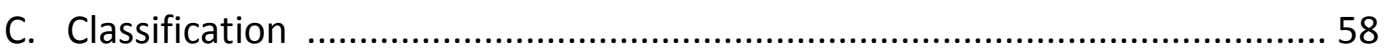

D. Generalization from the Supplemental Dataset ...................................... 59

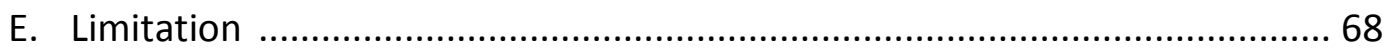

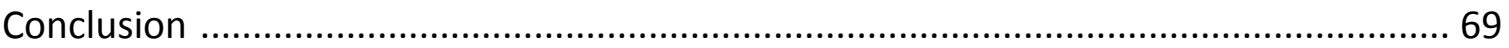

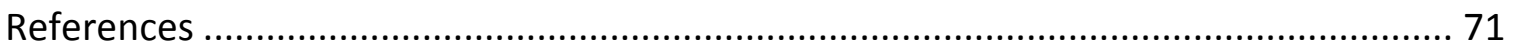

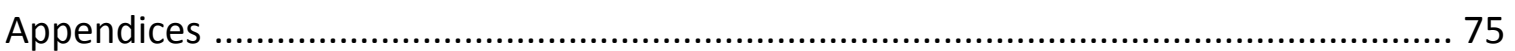

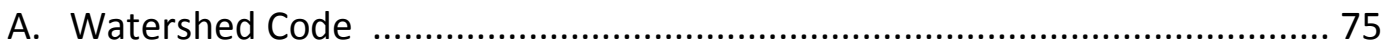

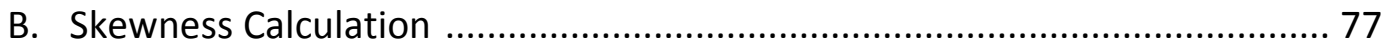

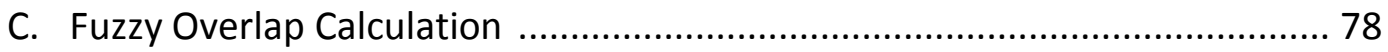




\section{$\underline{\text { List of Figures }}$}

Figure 1. Location of primary LiDAR datasets, acquired 22 July and 27 August 2010, respectively. ...... 14

Figure 2 Location of the supplemental LiDAR dataset, acquired in July 2008. 15

Figure 3 Standard false color composite of the digital ortho-image acquired at the same time as LiDAR data acquisition of the WVU organic farm July 2008upplemental dataset....... 16

Figure 4 Field Data overlaid on the canopy height model. .......................................................... 17

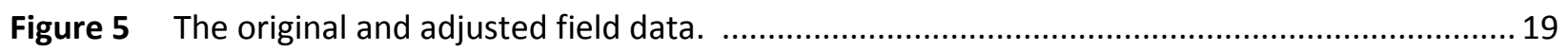

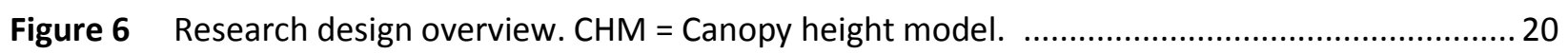

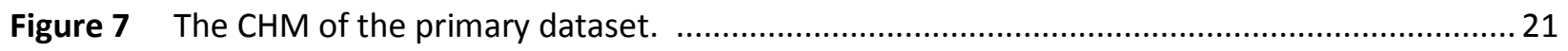

Figure 8 Comparison of trees. (a) Original IDW interpolation. (b) Adjusted IDW interpolation............ 22

Figure 9 Trees used to empirically set the segmentation settings. (a) Individual well-separated trees

(b) Row of trees (c) Clumped trees.

Figure 10 Comparison of the original and eroded field data, underlain by the watershed segmentation

Figure 11 The division of the field data into 5 different test sets. (a) Monongahela River and Falling Run area. (b) WVU Organic Farm area.

Figure 12 A comparison of the segmentation with the field data (a) TreeVaW segmentations (b) watershed segmentation.

Figure 13 A comparison of the classifications of the primary dataset (July 2010). (a)Watershed segmentation with CART. (b) TreeVaW segmentation with CART. (c) Watershed segmentation with NN. (d) TreeVaW segmentation with NN. (e) Watershed segmentation with SVM. (f) TreeVaW segmentation with SVM. (g) The extent of images $a-f$ in the CHM.

Figure 14 A box plot showing the STD of slope for Ailanthus, other deciduous and coniferous trees.... 49

Figure 15 A box plot showing the percentage of first returns for Ailanthus, other deciduous and coniferous trees 50

Figure 16 A box plot showing the percentage of intermediate returns for Ailanthus, other deciduous and coniferous trees. 
Figure 17 A box plot showing the maximum intensity for Ailanthus, other deciduous and coniferous trees.

Figure 18 The CHM model on the banks of the Monongahela River. The trees along the west edge of the image are well represented, but the elevated track of the Personal Rapid Transit (PRT) which runs down the center of the image, has only portions which were removed, while much of it was retained in the final CHM.

Figure 19 Under-representation of trees by TreeVaW segmentations (blue) compared with actual trees (white).

Figure 20 A comparison of the LiDAR point distribution between the August 2010 primary dataset and the supplemental July 2008 dataset.

Figure $21 \mathrm{CHM}$ of the test data where trees (white) are not fully outlined since the LiDAR points did not penetrate the crown and the canopy filter was not able to differentiate canopy from ground. This resulted in crescent-like trees. 62

Figure 22 Trees which were not included in the segmentation because the crown was not penetrated by the laser beam. The arrow indicates a tree missing in the CHM.

Figure 23 CHM with segmentation results superimposed using the supplemental July 2008 dataset. Interpolation artifacts which produces dimpled trees.

Figure $24 \mathrm{CHM}$ with overlain classified TreeVaW segmentation and associated field data undersegmentation of the supplemental July 2008 dataset. (a)Zoomed in view. (b) A view covering a larger area.

Figure 25 Under-segmentation of the supplemental dataset using watershed segmentation. 66

Figure 26 Over-segmentation and under-segmentation of the supplemental July 2008 dataset. (a) under-segmentation (b) over-segmentation.

Figure 27 Skewness calculation procedures.

Figure 28 Fuzzy segmentation accuracy assessment model in Imagine. 78 


\section{$\underline{\text { List of Tables }}$}

Table 1. The number of different trees measured in the field data used in the training of the classifier.

Table 2. List of attributes used to classify the LiDAR data.

Table 3. The accuracy of the TreeVaW and watershed segmentations for the primary and Supplemental datasets.

Table 4. Percentages of each tree species which were segmented correctly for July and August 2010, and July 2008, based on a sample of 78 Ailanthus, 64 Other Deciduous, and 33 Coniferous trees, respectively.

Table 5. Error matrix for the CART classification of the watershed segmentation calculated using trees which were correctly segmented. $\mathrm{A}=$ Ailanthus, $\mathrm{OD}=$ Other deciduous, $\mathrm{C}=$ Coniferous. Watershed segmentation (Values in the table represent number of trees $n=291,0.69$ overall accuracy)

Table 6. Error matrix for the CART classification of the TreeVaW segmentation calculated using trees which were correctly segmented. $\mathrm{A}=$ Ailanthus, $\mathrm{OD}=$ Other deciduous, $\mathrm{C}=$ Coniferous. TreeVaW segmentation (Values in the table represent number of trees $n=294$ and overall accuracy $=0.74)$.

Table 7. Error matrix for the NN classification of the watershed segmentation calculated using trees which were correctly segmented. $\mathrm{A}=$ Ailanthus, $\mathrm{OD}=$ Other deciduous, $\mathrm{C}=$ Coniferous. Watershed segmentation (Values in the table represent number of trees $n=291,0.54$ overall accuracy).

Table 8. Error matrix for the NN classification of the TreeVaW segmentation calculated using trees which were correctly segmented. $\mathrm{A}=$ Ailanthus, $\mathrm{OD}=$ Other deciduous, $\mathrm{C}=$ Coniferous. TreeVaW segmentation (Values in the table represent number of trees $n=294$ and overall accuracy $=0.62$ ).

Table 9. Error matrix for the SVM classification of the watershed segmentation calculated using trees which were correctly segmented. $\mathrm{A}=$ Ailanthus, $\mathrm{OD}=$ Other deciduous, $\mathrm{C}=$ Coniferous. Watershed segmentation (Values in the table represent number of trees $n=291,0.66$ overall accuracy.

Table 10. Error matrix for the SVM classification of the TreeVaW segmentation calculated using trees which were correctly segmented. $\mathrm{A}=$ Ailanthus, $\mathrm{OD}=$ Other deciduous, $\mathrm{C}=$ Coniferous. 
TreeVaW segmentation (Values in the table represent number of trees $n=294$ and overall accuracy $=0.58$ )

Table 11. Summary of the overall users' accuracy as the percentage of the classification based all trees in the field data.

Table 12. Summary of overall users' accuracy as the percentage of the classifications based upon trees which were correctly segmented.

Table 13. Attributes ranked by number of appearances in the CART decision trees. 46

Table 14. Attributes ranked by number of trees classified in the CART decision tree .48

Table 15. The formulas for the operations in the left side of the model in Figure 24 .79 


\section{ACKNOWLEDGEMENTS:}

I would like to thank West Virginia View for the financial support which allowed me to attend the ISPRS summer school. I would also like to thank Canaan Valley Institute and WVU NRAC who provided the LiDAR data, Adam Riley for helping me understand the LiDAR software, and for initially introducing me to the data. Dr. Conley and Dr. Landenberger have been greatly helpful during the progression of this thesis. Finally and most importantly, I would like to thank Dr. Timothy Warner who went above and beyond to help me complete this thesis. 


\section{CHAPTER ONE}

\section{INTRODUCTION}

The goal of this thesis is to compare the effectiveness of two image segmentation methods and three classification methods for delineating and differentiating Ailanthus altissima from other tree species using high point-density light detection and ranging (LiDAR, also written as LIDAR or lidar) data. LiDAR data have been used to delineate and classify individual trees (Brandtberg 2007; Orka et al. 2008; Reitberger et al. 2006) using LiDAR-based estimates of tree properties such as height, crown shape, and area, as well as laser characteristics such as the intensity of the returned pulse. Individual tree delineation and classification are related, complementary analytical tasks, as the accuracy of classification generally increases with the accuracy of the individual tree delineation (Brandtberg and Warner 2006). Ailanthus altissima, also known as the tree of heaven, is an invasive, exotic species, dominating many disturbed and urban areas in the United States, making it a concern for land managers in need of strategies in which to manage these plants (Craine 2009).

My first objective is to compare the effectiveness of two image segmentation methods: (1) a program named the Tree Variable Window program (TreeVaW), and (2) watershed segmentation. TreeVaW was developed by Dr. Sorin Popescu of Texas A \& M University and written in Interactive Data Language (IDL) (Popescu et al. 2002). TreeVaW uses a moving window which changes size and shape as it adjusts to individual tree height and crown shape. The program locates the apex of the tree by identifying the highest value within the moving window, and calculates the radius of the crown from that apex point based upon its height 
value using linear regression. The final product is a file of all the tree apices each with an associated height and crown radius (Popescu et al. 2003). The second segmentation method I use is watershed segmentation, which is based upon a raster canopy height model (CHM), a representation of the heights of individual trees and other objects on the ground surface after the topographic elevation has been removed. Watershed segmentation simulates filling the depressions in the inverted $\mathrm{CHM}$ with water. As the simulated water-level rises, the water in the individual depressions merge, and the line between the merging basins outlines the individual segments, which are assumed to represent, in this case, tree crowns (Mangan and Whitaker, 1999).

My second objective is to evaluate the potential for differentiating Ailanthus altissima from other tree species using LiDAR-derived attributes such as return distribution with height and intensity of pulse return. I compare the effectiveness in classification using these metrics provided by LiDAR data in three different classification methods: classification and regression trees (CART), support vector machines (SVM) and artificial neural networks (NN).

CART creates a decision tree, which is a classification procedure for organizing individuals into divergent classes based on a series of similarities and differences. The data is split repeatedly until all of the data is organized into classes (De'ath and Fabricius 2000).

SVM is a supervised learning method, which predicts what class the input value belongs to by comparing two classes at a time. The data are projected from an initial smaller number of dimensions into a hyper-dimensional space such that the overlap between the classes is 
minimized. Because SVM is searching through so many additional dimensions, the classification decision boundaries may be difficult to understand (Melgani and Bruzzone 2004).

NN classifiers are information processing systems inspired by the structure of biological neurons in the brain. The network of neurons takes the input data and produces an output based on the knowledge that the neurons have "learned" by adjusting weights in the network that categorize the outputs into the assigned classes (Bischof et al. 1992). 


\section{CHAPTER TWO}

\section{LITERATURE REVIEW}

In this section I will cover background on Ailanthus altissima, LiDAR data, image segmentation methods, and tree classification methods which use LiDAR.

\section{$\underline{\text { Ailanthus altissima }}$}

Considered a weedy species, Ailanthus altissima is a dioecious deciduous tree in the Quassia family (Simaroubaceae) native to the temperate forest of central China (Kowarik 1995). Ailanthus altissima was originally planted in the US in the $19^{\text {th }}$ and $20^{\text {th }}$ centuries as a decorative tree, because of its resilience in the urban environment. It spread because of its tolerance of infertile, compacted soils, which promoted rapid growth and early maturity, making it one of the most aggressively spreading invasive species in newly disturbed areas. Mature females produce up to 300,000 anemochorous seeds which are dispersed from late summer to late spring (Kowarik 1995). The species tends to shade out its slower growing competitors (Landenberger and Kota 2006). Because it is a light-demanding plant, the structure of its canopy tends to be umbrella-shaped so that it shades the competing plants below, which are therefore only able to grow more slowly (Craine 2009).

Since Ailanthus altissima is a shade-intolerant species, it is not generally identified as an invader of intact forests, but it has been observed to colonize natural and anthropogenic gaps in forests. Within gaps, the height, diameter and crown radius of the tallest Ailanthus sapling tend to be greater than those of the tallest native competitors (Canham and Knapp 2000). 
The problem with Ailanthus species from an economic perspective is that the rapidgrowth due to its low light strategy causes the stem to be of low commercial quality, not comparable to the native species which the Ailanthus out-competes. From an ecological perspective, Ailanthus displaces trees upon which the local biota may have evolved to become dependent (Craine 2009).

\section{$\underline{\text { LiDAR }}$}

LiDAR is an active remote sensing technology often used in forestry inventory. A raw LiDAR dataset comprises a collection of points in three-dimensional space and is known as a point cloud. Each LiDAR point represents the location of the surface that reflected the laser beam and has associated attributes, including $X, Y, Z$ coordinates, intensity, range, and return number. The location of each LiDAR point is determined by measuring the time it takes for the laser beam to reflect back to the sensor from which it was generated. The travel time is used to estimate the distance to the point, in conjunction with an estimate of the location and orientation of the originating sensor using an on-board global positioning system (GPS) and an inertial navigation system. LiDAR data acquired from aircraft (known as range finder LiDAR) typically are generated with a fine laser beam, and a scanned pattern which makes it well suited to model tree canopies (Palleja et al. 2010 ; Leigh et al. 2009). Each outgoing laser pulse can produce multiple returns, allowing for not just the upper canopy surface, but also potentially the vegetation structure and even the understory and the ground, to be identified (Brandtberg 2007). Filters for differentiating these points and are commonly used to separate the different layers of LiDAR data into ground points, first returns, last returns, and other combinations of points. In 
forestry applications, two particularly useful layers are the bare earth layer, which consists of a combination of first-and-only and last returns, and the first return layer, which consists of only first returns (Shan and Toth 2008).

LiDAR data may include a record of the intensity of the reflected pulse. Intensity data may be useful for classification, since different physical structures will reflect the laser pulse differently, and potentially even indicate species difference (Mitasova et al. 2005; Brandtberg 2007). LiDAR data can be used to estimate the heights of trees better than any other non fieldbased method. However, the most precise heights of a tree is difficult to get since it is likely that the apex of the tree will be missed by a laser, so the higher the LiDAR point density the better (Falkowski et al. 2009). LiDAR points are usually also generated from within the forest canopy, making it possible to estimate the leafy biomass, which may be used as yet another species indicator (Popescu et al. 2009). Sometimes LiDAR data is kept in this point format. For instance, when tree classification using tree profile points is undertaken, the three dimensional tree is subdivided into vertical segments so that structural attributes derived from the individual points can be used in classification algorithms (Brandtberg 2007). Because LiDAR data is dependent upon physical interactions of the laser and the surface of the reflecting object, the time of the year of data acquisition is an important factor in determining vegetation metrics for deciduous species; during leaf-on periods branches and leaves generate LiDAR returns, whereas in the leaf-off periods, only the branches and seed pods generate returns (Asner et al. 2008).

LiDAR data can be transformed from a point value format to a raster value format, potentially making it easier to analyze the data using raster-based remote sensing software. Raster-based pixel values is the default data structure for most image-processing software 
packages, and most remote sensing software packages are limited both in functionality for point data and in capacity for dealing with the generally large numbers of points. However, rasterization needs to be carried out with caution, as a raster grid that is too coarse can mask the patterns, whilst a grid that is too fine requires excessive interpolation. In addition, raster grids generally obscure the complexity of multiple returns from a single outgoing laser pulse unless multiple raster datasets are produced.

\section{$\underline{\text { Segmentation Methods }}$}

Segmentation is the dividing of an image into useful subareas. If these subareas represent the individual trees, this potentially makes it possible to measure the crown shape, height, diameter, and other factors which may be useful indicators of the species. Without identifying individual trees, it is difficult to do classification on anything other than the individual points or pixels (Popescu et al. 2003).

There are many types of image segmentation methods that can be used to separate individual trees in raster data, including blob detection (Brandtberg et al. 2003), canopy maxima modeling, watershed segmentation, and valley-following tree delineation (Ham et al. 2005). Conceptually, most methods are based on the assumption that individual trees are represented in the LiDAR data by regions of higher elevation surrounded by regions of lower elevation. These methods are predominantly derived from theories in computer vision. Most segmentation methods involving tree separation identify local minima (lowest values) or maxima (highest values) within a defined area as a way to extract the unique tree shape and best separate the trees into individual segments. The local maxima usually represent the apex 
of the tree, and the local minima are usually used to locate the valleys between tree crowns. Coniferous trees are generally more easily identified than deciduous trees due to the approximately conical shape of coniferous crowns compared to the complex asymmetrical shape of deciduous crowns (Bork and Su 2007). Popescu et al. (2003) found that for the tree delineation program TreeVaW, circular windows are better for deciduous trees and square windows are better for coniferous tree identification. Indeed, the window in TreeVaW is variable in shape, specifically to facilitate mapping mixed forests. The variable window method is similar to the canopy local maxima model, but is more effective at delineating trees with complex structure such as deciduous tree species, more often separating even the more complex, non-symmetrical deciduous tree crowns into complete units.

Watershed segmentation has the benefit of being simple to use and parameterize, and is commonly included within commercial software packages, such as ENVI (Exelis, Boulder), TOPAZ (University of Saskatchewan, Saskatoon) and IDRISI (Clark Labs, Worcester). It also handles deciduous trees better than other methods since it is much more reliant upon the crowns' three-dimensional shape for the delineation calculations. However, deciduous trees are typically flat-canopied, which makes it more difficult to place the valley detection line which separates the crowns, compared to coniferous trees which usually have a detectable valley between trees (Ham et al. 2005). Therefore, over-segmentation, or splitting the trees into many segments, is common with deciduous tree crowns (Mangan and Whitaker 1999). Gaussian filtering is commonly used to smooth the tree canopy model prior to analysis, suppressing gaps 
and generalizing the shape, a step that generally increases the accuracy of deciduous tree segmentation (Mangan and Whitaker 1999).

\section{$\underline{\text { Classification }}$}

LiDAR provides potential insights into the trees' canopy structure which no alternative remotely sensed data can provide. Since returns are generated by the upper surface of the forest canopy, as well as from within the canopy, the entire three-dimensional profile of the trees can be modeled as well as the distribution of the biomass within the canopy (Popescu et al. 2009).

Numerous summary attributes have been generated from LiDAR data for classifying forest species, including crown height, width, height of leaf canopy, thickness of branches, and reflectance of foliage (Brandtberg 2007). The most useful measures of individual trees in species classification are usually tree height, crown diameter, and mean intensity of reflectance values (Orka et al. 2008). The highest accuracy rates for species identification using only LiDAR have varied from 65\% (Brandtberg 2007) to 75\% (Queen et al. 2009). Multispectral and hyperspectral studies using visible, near infrared and shortwave infrared have been used to classify tree species by identifying differences that may be associated with foliar chemistry. These classifications of forest cover types such as conifer, deciduous, and mixed stands have classifications of up to $75 \%$ accuracy (Martin et al. 1998). Combined multi-spectral and LiDAR classifications have also been used in species identification, yielding classification accuracies as high as 96\% (Holmgren, et al. 2008). 
A LiDAR attribute of great potential use for species classification is the intensity of the returned pulse. Most LiDAR systems use a laser in the near infrared, at approximately $0.9 \mu \mathrm{m}, \mathrm{a}$ region in which leaves have little absorption, but strong reflectance and transmittance (Brandtberg 2007; Song et al. 2002). The intensity values are also dependent on the size of the object relative to the laser pulse beam width; for example, larger branches or leaves generally return a greater proportion of the laser pulse than smaller branches or leaves (Morsdorf et al. 2009).

Intensity values are generally not calibrated, and thus their use is effectively limited to comparisons within a single data set. Nevertheless, relative intensity can be useful for differentiating vegetation from other surface materials (Mitasova et al. 2005), and potentially even identifying tree species using attributes such as mean, maximum, and minimum intensity (Orka et al. 2008).

For a single outgoing laser pulse, each successive return reduces the remaining ongoing energy, and thus the intensity of later returns will also tend to decrease (Brandtberg et al. 2003). Thus, Brandtberg (2007) cautions against combining intensity data from first and subsequent returns. For this reason, intensity from the canopy surface, generally comprising first returns, is regarded as the most useful attribute for characterizing features.

Determining the optimal classification scheme may depend in part upon the attributes of the LiDAR data that are collected. NN (Bischof et al. 1992), SVM (Heikkinen et al. 2010) and CART algorithms (Ham et al. 2005) have been used to classify multispectral images of forests. NN have been used in tree classification of species data, but have not yet, to my knowledge, 
been utilized with LiDAR derived attributes for tree species classification. SVM has been used to classify vegetation species using LiDAR data, and is one of the leading methods in threedimensional object recognition which makes it suitable for classification of LiDAR data (Bruzzone and Melgani 2004).

For the CART program, each splitting point in a desired classification tree is called a node. Each node has one parent node (except for the root), although it can have multiple descendant nodes. These splits are determined, or "learned," using the training data provided to the CART program. Therefore, the training data must be of high quality for an adequate classification (Bodley and Friedl 1997). CART is a commonly used method with complex ecological data, where large amounts of information are used to determine classes based upon the attributes which the classes share. This method of classification is very flexible regarding data type, and able to handle both numerical and categorical data. CART can also handle nonlinear relationships, or incomplete datasets (De'ath and Fabricius 2000). CART commonly over-fits the data, categorizing all data in an attempt at $100 \%$ classification (Lawrence and Wright 2001), and therefore the decision tree should be "pruned" to compensate for this overfitting (Bodley and Friedl 1997).

NN are non-linear systems, having statistical components in the algorithm design. The neurons "learn" by adjusting the relationships within the data, and categorize the output into appropriate classes based on what was "learned." NN are generally regarded as being robust in the presence of noisy data and having the ability to handle irregular datasets (Bischof et al. 1992). NN often produce a better classification than maximum likelihood classification, a 
method that is usually regarded as the standard. NN also very easily incorporate other physical features such as texture into the classification (Bischof et al. 1992).

SVMs have been found to classify multispectral imagery more accurately than both maximum likelihood and NN classification. The classes are differentiated by projecting the data into a higher dimensional space that best separates the support vectors, or training data, on the boundary of the classes. SVM focuses on generating boundaries between two classes rather than finding the similarities within each potential class. It is very useful in remote sensing when multiple attributes are used for classification, but does also have a number of user-specified parameters (Melgani and Bruzzone et al. 2009). 


\section{CHAPTER THREE}

\section{$\underline{\text { METHODS }}$}

\section{Study Site and Data}

The study sites are located in the area around Morgantown, West Virginia. Morgantown has a variety of habitats where Ailanthus altissima thrives, including urban development and disturbed forest. Three LiDAR high point density datasets were obtained. I used the first two datasets, which I term the primary datasets, to develop and test my methods. In order to evaluate the robustness of the findings, I also tested my methods with a third dataset, which I call the supplemental dataset.

The two primary LiDAR datasets were acquired on 22 July 2010, and 27 August 2010, respectively (Figure 1). Both primary datasets were collected by the West Virginia University Natural Resource Analysis Center (NRAC) using an Optech ALTM 3000 LiDAR system. The flight height for the acquisition was approximately $600 \mathrm{~m}$, with an average flying speed of 135 knots. The pulse rate was $100 \mathrm{KHz}$, with a scan frequency of $50 \mathrm{~Hz}$ and a maximum scan angle of $24^{\circ}$. Up to four returns were recorded for each outgoing laser pulse. The point density is relatively high, with 10.3 points $/ \mathrm{m}^{2}$. 


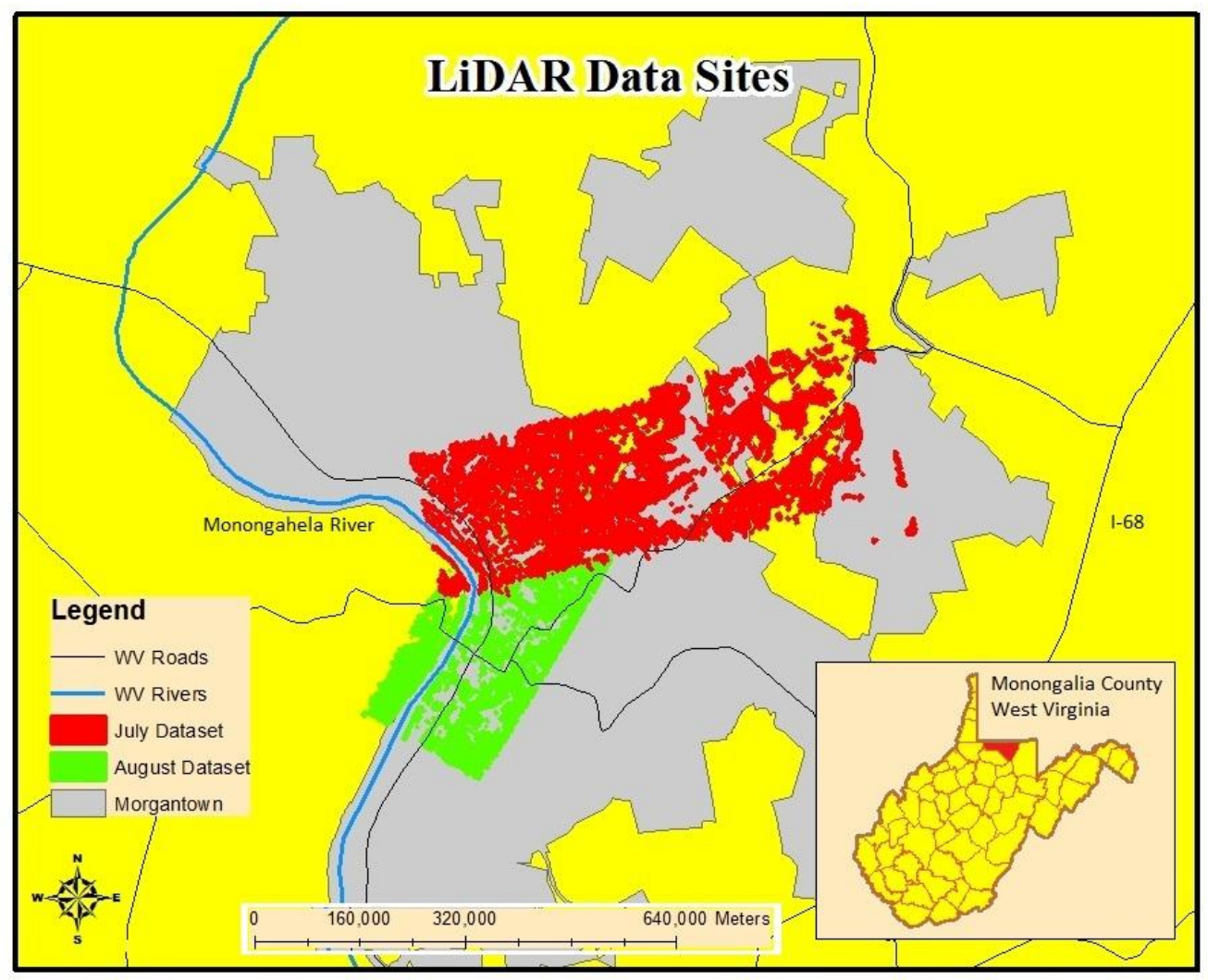

Figure 1: Location of primary LiDAR datasets, acquired 22 July and 27 August 2010, respectively.

The third and supplemental LiDAR dataset (Figure 2), was acquired in 2008 with associated orthoimages by the Canaan Valley Institute (CVI) using the same Optech ALTM 3000 system as the NRAC data. These data were acquired with a maximum $20^{\circ}$ scan angle, a scan frequency of $40 \mathrm{~Hz}, 70 \mathrm{kHz}$ pulse rate producing 4.7 points $/ \mathrm{m}^{2}$, and from an altitude of 1,200 $\mathrm{m}$ above the ground. The third dataset was acquired over two regions: the WVU organic farm (Figure 3), and along the banks of the Monongahela River, and the adjacent rail-trail. 
I chose to include the supplemental dataset because the two locations overlapped with my field data for the primary dataset. The two July 2008 areas in the supplemental dataset are comparatively small, and were originally chosen for data collection because they are areas of known occurrences of Ailanthus altissima. These supplemental datasets cover intact and fragmented forests, farms, and urban clusters of growth. This allowed for a relatively comprehensive test of mapping Ailanthus in different environments, with data acquired at different times, and at different point densities.

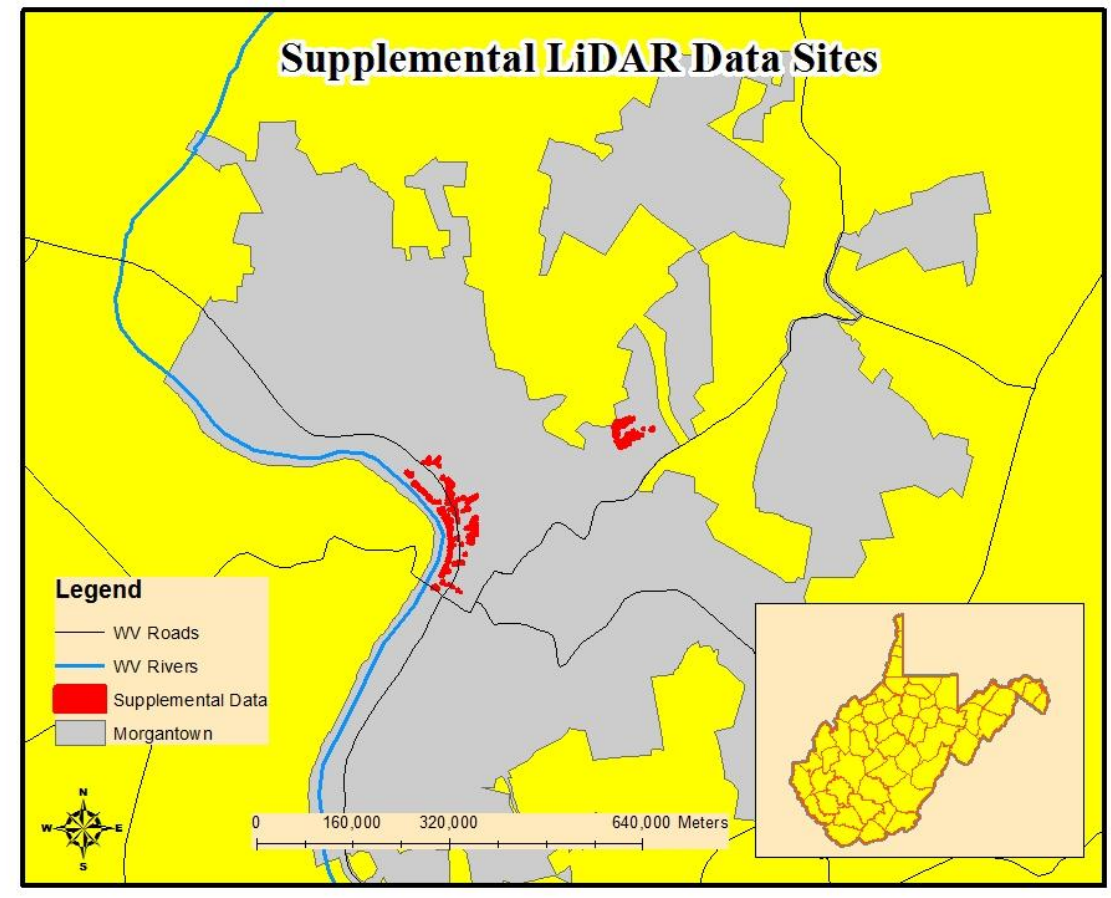

Figure 2: Location of the supplemental LiDAR dataset, acquired in July 2008. 


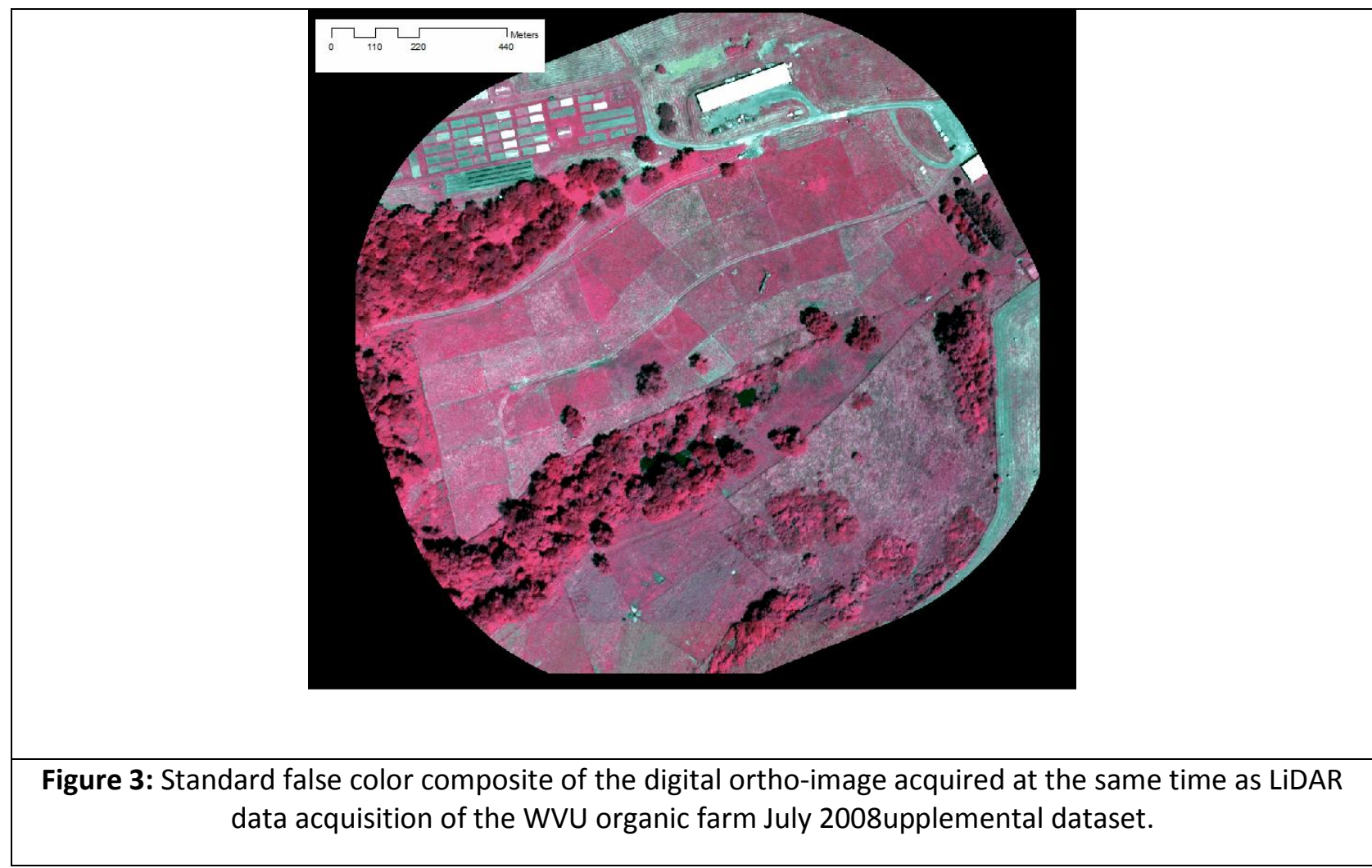

\section{$\underline{\text { Field Data }}$}

I selected a small portion of the study site to identify locations of individual trees, for training and evaluating the results of my classification. The field data locations were along the Monongahela River, within the WVU campus, around Falling Run Road, and within the WVU organic farm (Figure 4). I collected a total of 175 tree samples (Table 1) using a Magellan Mobile Mapper Global Positioning System (GPS). For each tree both the center and the edge of the crown was mapped. 


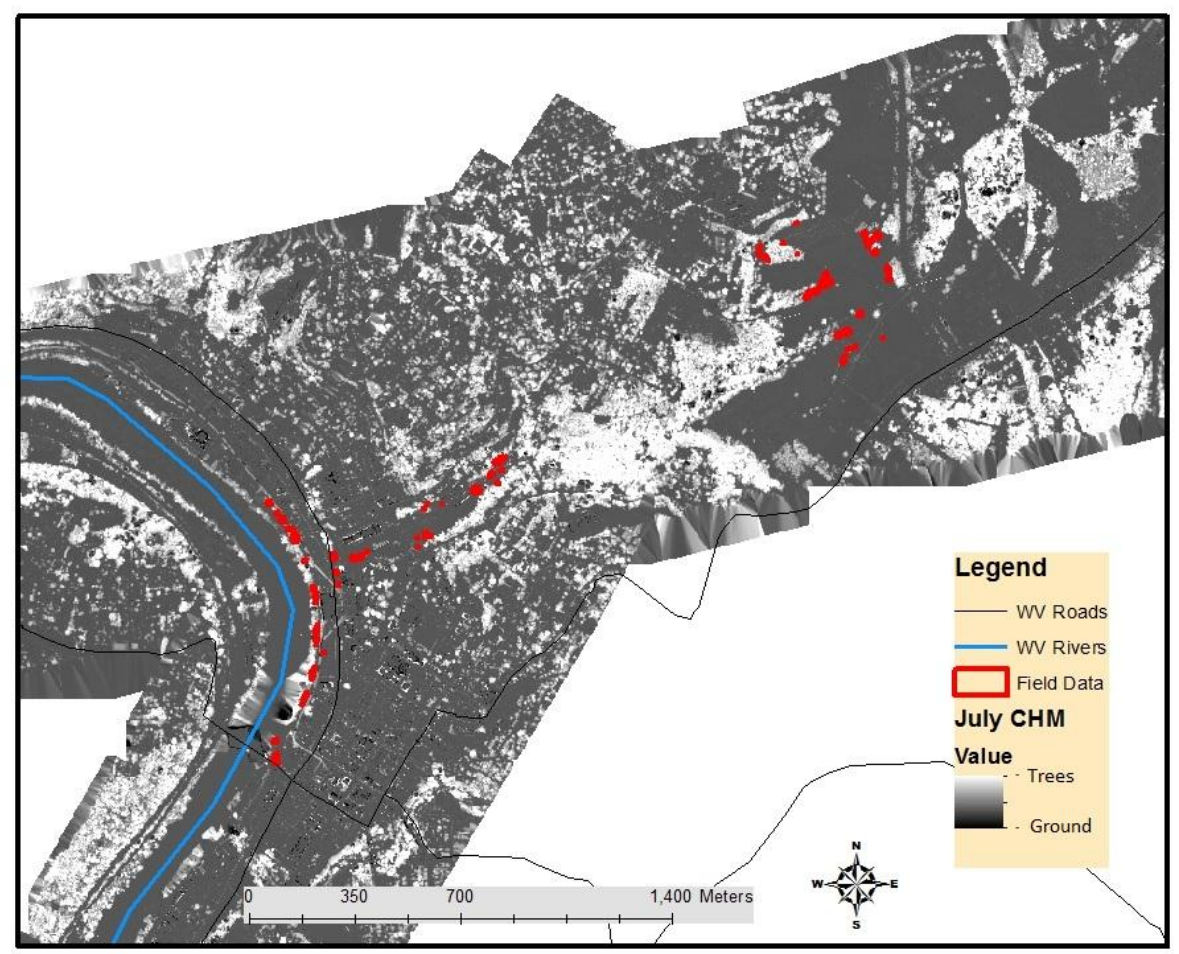

Figure 4: Field Data overlaid on the canopy height model.

\begin{tabular}{|c|c|c|c|c|}
\hline Table 1: The number of different trees measured in the field data used in the training of the classifier. \\
\hline Class & $\begin{array}{c}\text { Number used in } \\
\text { calibration of the } \\
\text { segmentation of } \\
\text { primary dataset }\end{array}$ & $\begin{array}{c}\text { Number used in } \\
\text { training the } \\
\text { classification of } \\
\text { primary dataset }\end{array}$ & $\begin{array}{c}\text { Number used in } \\
\text { calibration of the } \\
\text { segmentation of } \\
\text { supplemental } \\
\text { dataset }\end{array}$ & $\begin{array}{c}\text { Number used in } \\
\text { training the } \\
\text { classification of } \\
\text { the supplemental } \\
\text { dataset }\end{array}$ \\
\hline Ailanthus altissima & 78 & 105 & 78 & 39 \\
\hline Other Deciduous & 64 & 94 & 64 & 49 \\
\hline Coniferous & 33 & 57 & 33 & 0 \\
\hline
\end{tabular}

I collected the field data in October 2011. The LiDAR data from the primary dataset was collected in July and August 2010, and the supplemental data was collected in July 2008 . These differences in dates will cause a slight discrepancy in the segmentation accuracy, since the tree crowns may have changed in shape between the LiDAR acquisition time, and my field data 
acquisition time. Fortunately, the relative difference in the crown shape and diameter will be greatest in very small trees which are not likely to appear in the CHM in the first place (Craine, 2009).

The accuracy of the field GPS delineations of the tree canopy and the trunk locations varied with the GPS signal quality, which is primarily a function of the proportion of the sky in view and the geometry of the satellites at the acquisition time (Dussault et al. 2001). The field data were acquired with a GPS system which had a nominal $2 \mathrm{~m}$ error. In experiments in which I repeated tree crown mapping, in order to test for the precision of the mapping, I found a maximum error of $4 \mathrm{~m}$, in situations where the trees had very thick canopies, and in areas near large obstructions such as buildings or other obstacles. To try to minimize this error, I printed several images of the tree crown outlines overlaid on the intensity image. I then returned to the field, and determined how to manually shift the polygons so that they were better aligned with the LiDAR data. In many cases the error was consistent over a large region, lending further credence to the correction approach adopted (Figure 5). 


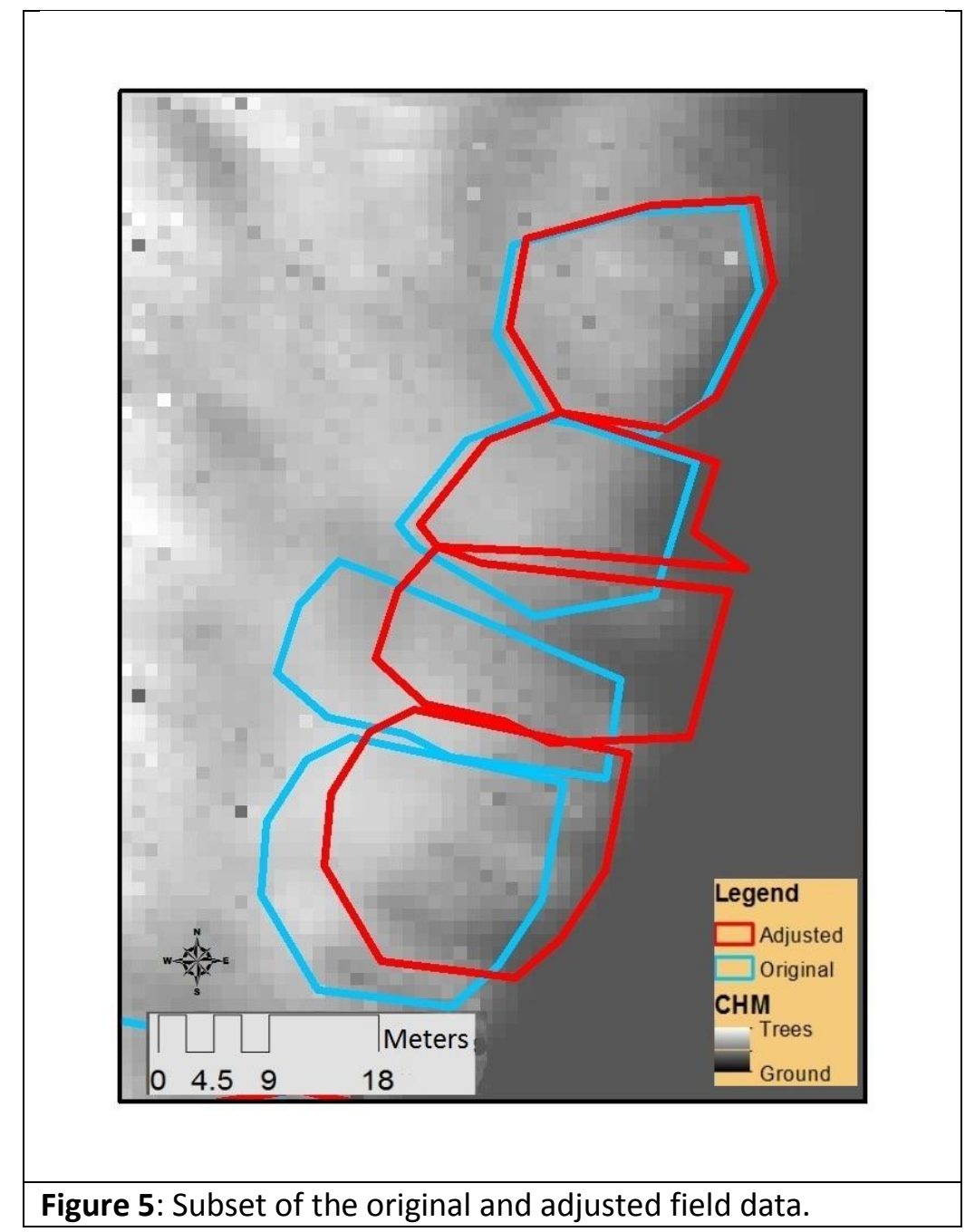

\section{$\underline{\text { LiDAR Analysis }}$}

My research aims focused on both segmentation and classification of Ailanthus trees. The combination of these two aims resulted in 4 image segmentations and 6 classifications (Figure 6). The 4 segmentations are created to compare the effectiveness of the watershed and TreeVaW segmentations on both the primary and supplemental CHMs. The 6 classifications were created so that I could compare the accuracy of the tree classification methods with each segmentation. 


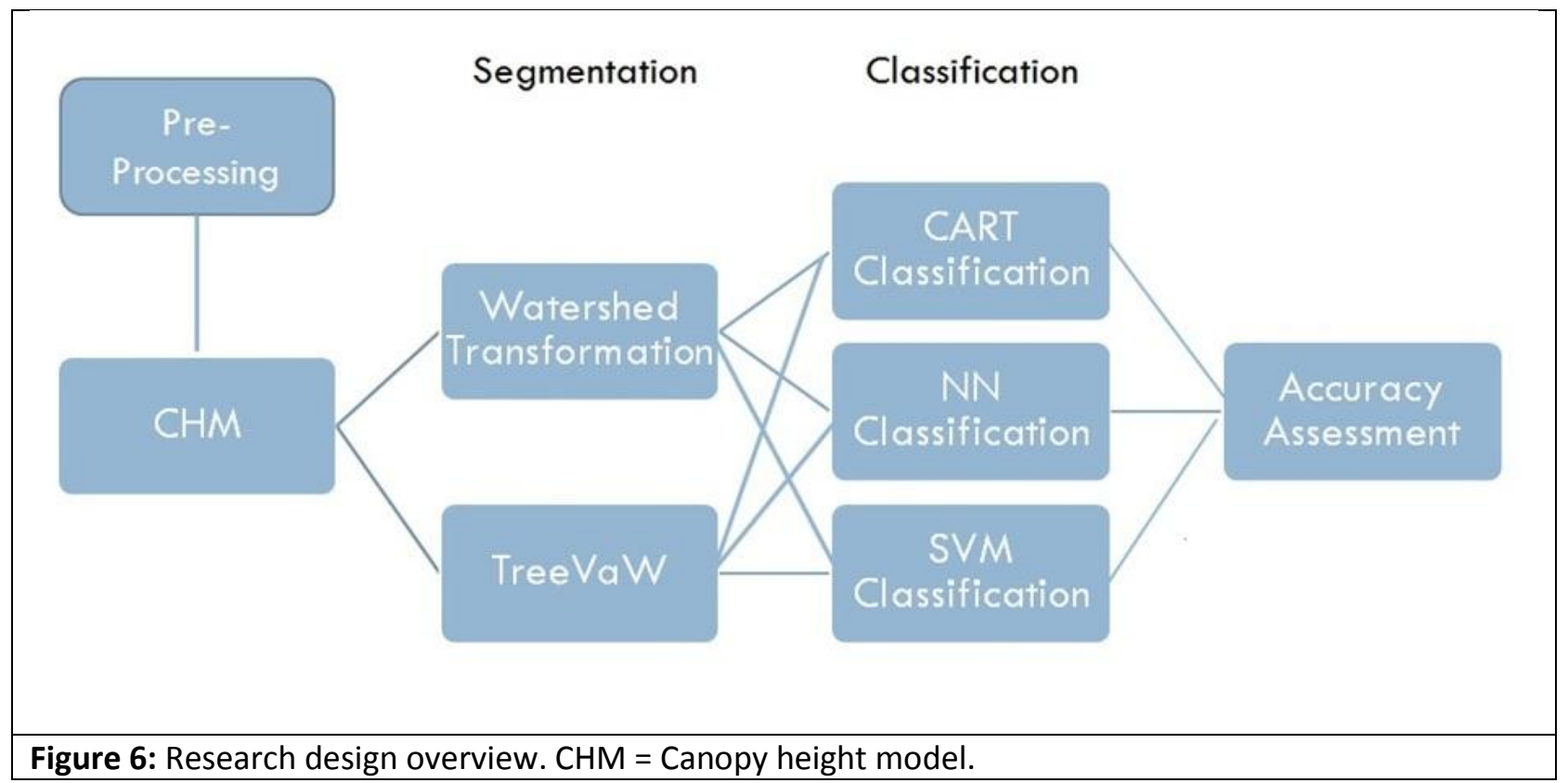

I began the remote sensing analysis by separating the LiDAR data into ground and canopy layers, with points labeled as vegetation and non-vegetation using the Terrascan extension to the Bentley Microstation software (Terrasolid, Helsinki), and the "Define classes by return number" function. Points which were first and only returns, or last returns, were classified as ground, and points which were first of many or had multiple returns were classified as canopy points. The bare-earth points were already classified for the preliminary dataset, so I used a "Detect building filter" for classifying buildings which I added in the ground layer. The building detection filter classifies points as buildings based on the return number, elevation and the distance between each return in an attempt to match these potential building returns to a defined set of building metrics. 


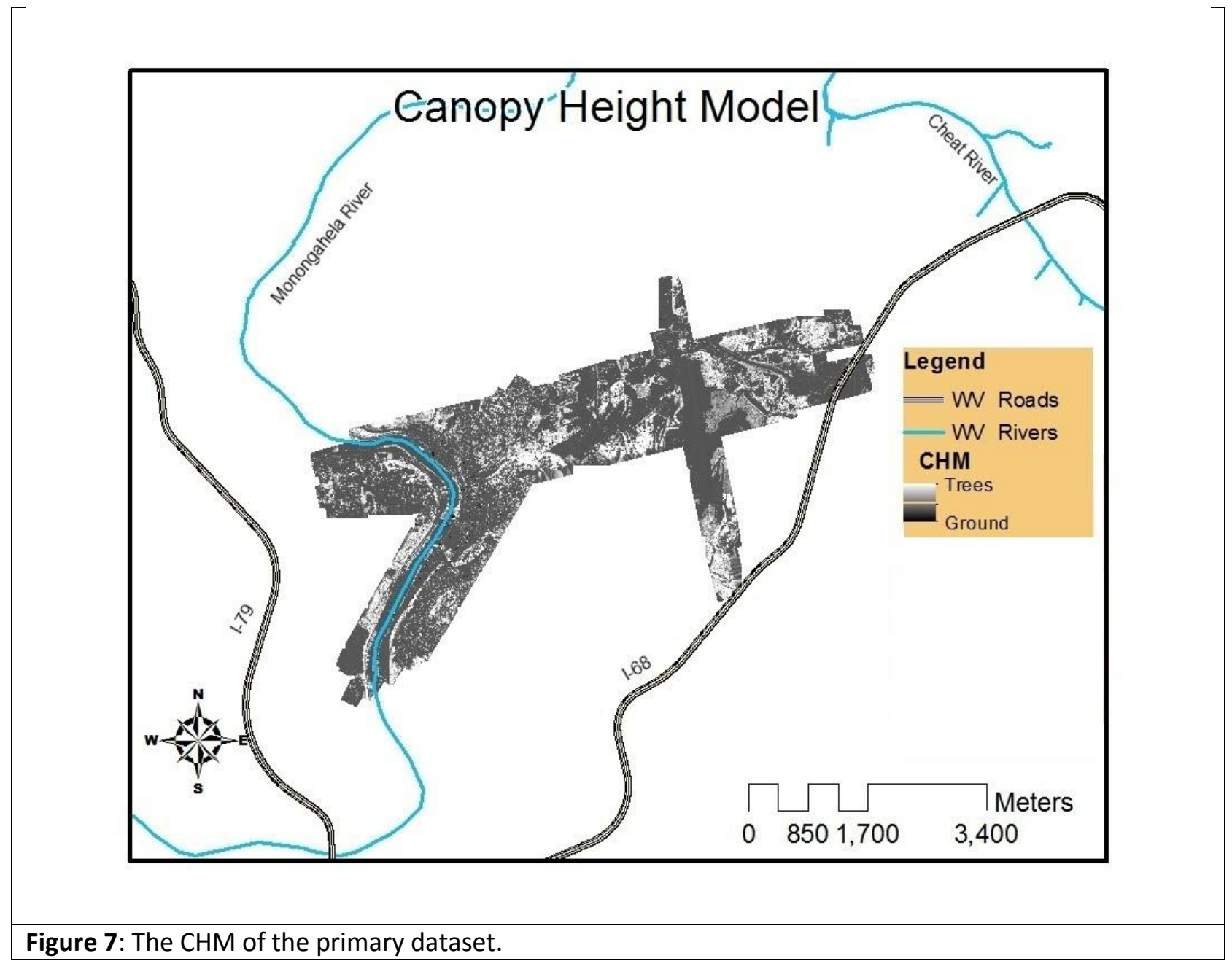

Next, I created a CHM with 0.25 m pixels (Figure 7). To generate the CHM, I first had to create a Digital Elevation Model (DEM) which is a raster image of the bare ground, with trees, buildings and other objects removed, and a Digital Surface Model (DSM), which is a raster dataset of the highest points in elevation including bare ground and tree tops. The first step in CHM creation is to import the .Las format point cloud LiDAR file as a multipoint coverage into ArcGIS. The interpolation method I chose for the surface and ground layers was Inverse Distance Weighting (IDW), using a power of 1 , and including 70 neighbors, in an attempt to 
minimize the linear features which often appear in interpolated LiDAR data because of the sweeping motion of the LiDAR sensor (Figure 8). In IDW, each interpolated value includes several immediate neighbors in determining influence on the interpolated units (Myers 1994). Interpolating the multipoint values from the first returns creates a DSM, and interpolation of the last returns creates a DEM. The DEM is then subtracted from the DSM using the raster calculator in ArcGIS, creating the CHM. The output is a raster dataset with the normalized heights of each tree, and the topographic elevation removed.
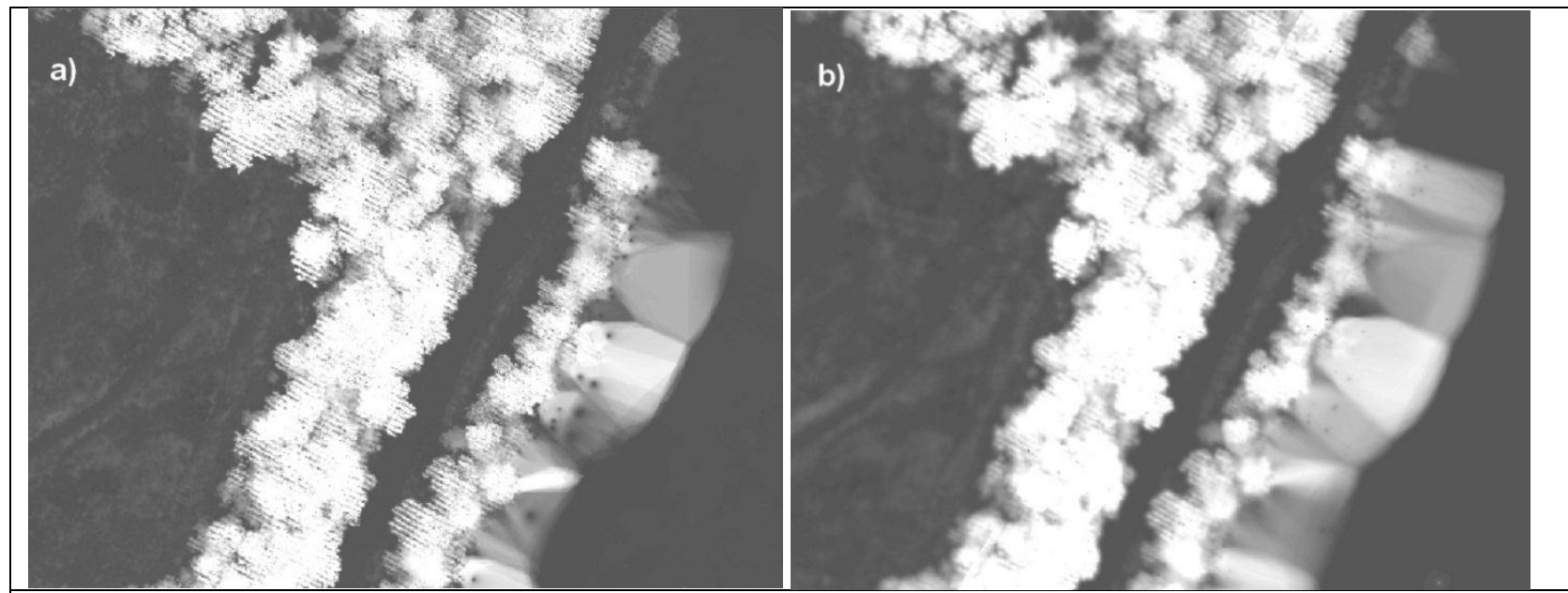

Figure 8: Comparison of trees. (a) Original IDW interpolation. (b) Adjusted IDW interpolation with striping reduced.

The tree delineation was then carried out. The watershed segmentation employed a custom program developed from a script provided in the IDL help (Appendix 1), and the TreeVaW segmentation program (Popescu et al. 2002), also written in IDL, was obtained from http://ssl.tamu.edu/personnel/s_popescu/treevaw.

The watershed segmentation program utilizes mathematical morphology (IDL Online Help 2011). The program was modified so that it could be used to select trees as watersheds on a 
CHM. The outcome of the watershed segmentation program was a new vector dataset with segmented polygons, assumed to represent tree crowns. The major user-specified parameters in the watershed segmentation were the size of the low-pass Gaussian filter used for smoothing, search radius of the structuring element, and the level of neighbor connectivity. The values I used for these parameters were $15 \times 15,35$ and 8 , respectively. The values were chosen empirically, through testing a variety of combinations of parameters until I obtained what appeared to be optimal results for individual trees, clumped trees, and a row of trees (Figure 9).
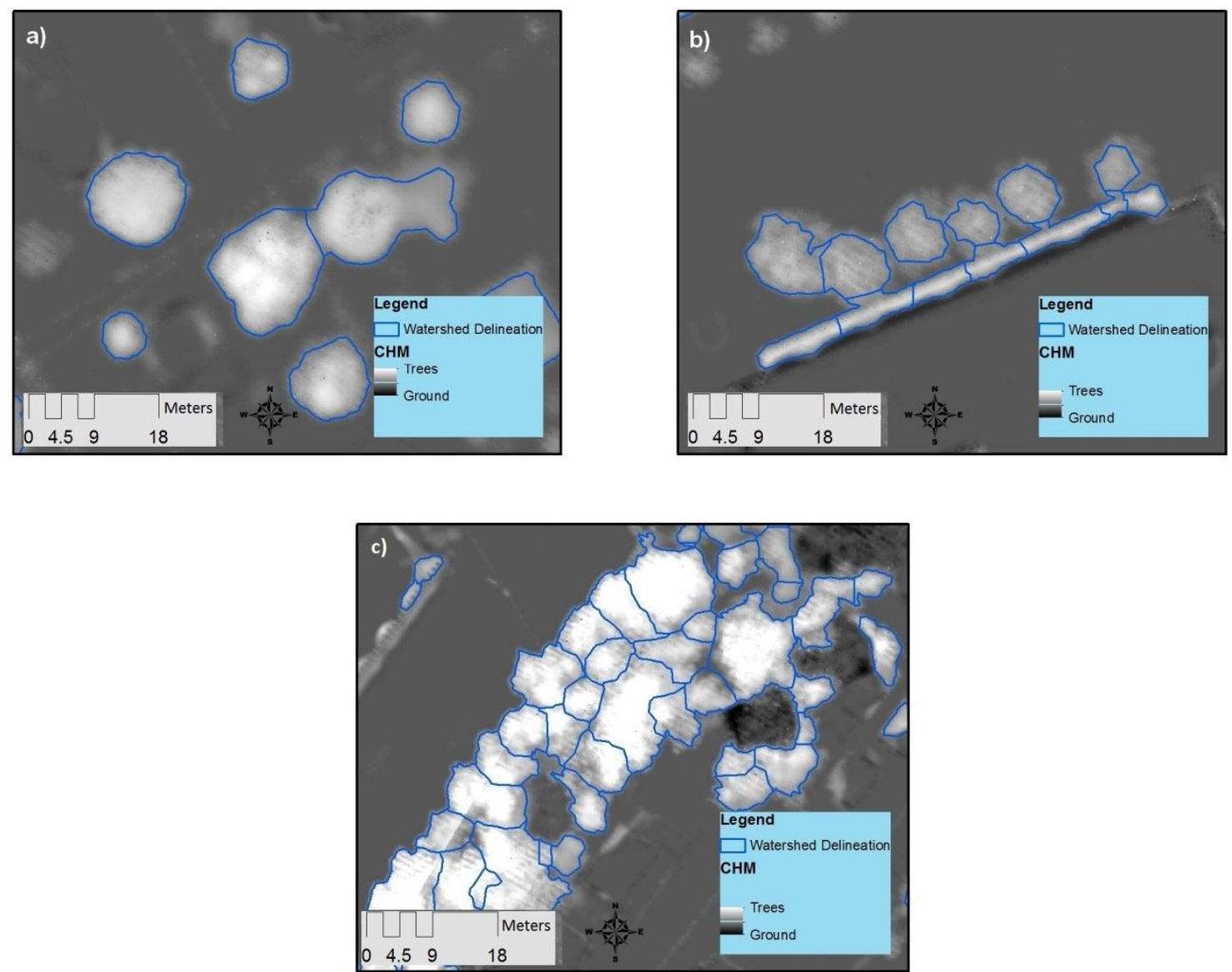

Figure 9: Trees used to empirically set the segmentation settings. (a) Individual well-separated trees (b) Row of trees (c) Clumped trees. 
The derived watersheds were originally generated as bitmap images, and then imported into Imagine software (Intergraph, Norcross), where the polygons were extracted, so that the watersheds were each given a unique identification number and the polygons that were smaller than 175 pixels were eliminated. Border pixels that defined the boundary of each watershed were also eliminated and assigned the values of the adjacent watershed. The final collection of watersheds representing individual trees was then imported into ArcGIS and a coordinate system assigned.

Watershed segmentation works in a straight-forward manner when a tree is surrounded by other trees. However, when a tree is isolated, each tree is not individually delineated, instead a single watershed boundary is generated between each pair of trees. Therefore, to exclude the exposed ground areas between trees, and focus on just the region of the canopy extent, I ran a custom process tree in eCognition (Trimble, Sunnyvale) designed to separate trees from bare ground. The process was developed by Jarlath O'Neil-Dunne, a geospatial analyst with the University of Vermont's Spatial Analysis Laboratory. His process tree entitled "Lidar Trees," identifies the vegetation, allowing the exposed ground to be masked out. This forces the edges of each watershed to the edge of the canopy.

The TreeVaW software locates the apex of each tree and assigns it a diameter value. By adding buffers around each point according to the diameter value, tree crowns can be defined using a fuzzy boundary. These buffers are overlain over the $\mathrm{CHM}$ in order to extract heights and other attribute values within the crown of each tree. 
The most important factor affecting the TreeVaW output was the amount of smoothing of the CHM. The optimal TreeVaW segmentation settings were chosen empirically based upon comparison with the field data. The smoothing was evaluated based on both the number of crowns identified and the area of these crowns. Generally, Gaussian smoothing filters smaller than $9 \times 9$ produced gross over-segmentation, and anything larger than $17 \times 17$ caused undersegmentation. The final smoothing setting selected was a Gaussian 15x15 filter.

Attributes for classification of the trees were derived from each polygon of LiDAR point data, as well as the CHM and DSM (Table 2). The attributes which I chose were based upon previous studies. In addition, I developed a new attribute, crown slope, which I added to more easily separate conical crowns of the coniferous trees from complex crowns of the deciduous trees. Crown slope is calculated by running the "slope" program in the ArcGIS Spatial Analyst toolbox indicating the percent of slope, on the original (non-smoothed) CHM, to get the percent slope of the outer surface of the tree crowns. The main method of calculating the attributes for each tree was by using the 'zonal statistics' tool in ArcGIS (ESRI, Redlands), supplemented by custom scripts.

The height attributes were derived from the CHM and the DSM so that heights and number of LiDAR returns at each quartile could be generated. Intensity values were only used from the first returns (Brandtberg, 2007). 


\begin{tabular}{|c|c|c|c|}
\hline Type & Description & Measure & Cited \\
\hline \multirow[t]{3}{*}{ Height } & Tree height (raw) & $\begin{array}{l}\text { maximum, range, mean, standard deviation, } \\
\text { sum, median, coefficient of variation }\end{array}$ & Donoghue et al. 2007 \\
\hline & $\begin{array}{l}\text { Tree height (normalized by } \\
\text { maximum height) }\end{array}$ & $\begin{array}{l}\text { minimum, range, mean, standard deviation, } \\
\text { sum, median, coefficient of variation }\end{array}$ & Kin et al. 2011 \\
\hline & $\begin{array}{l}\text { Relative }\left(25^{\text {th }}, 50^{\text {th }}, 75^{\text {th }} \text { and }\right. \\
90^{\text {th }} \text { percentiles) as } \\
\text { proportional to maximum } \\
\text { height }\end{array}$ & Height (normalized), & Kin et al. 2011 \\
\hline Intensity & $\begin{array}{l}\text { Intensity values for crown } \\
\text { using first returns }\end{array}$ & $\begin{array}{l}\text { minimum, maximum, range, mean, standard } \\
\text { deviation, sum, median, coefficient of } \\
\text { variation }\end{array}$ & Kin et al. 2011 \\
\hline \multirow[t]{4}{*}{$\begin{array}{l}\text { Tree } \\
\text { Shape }\end{array}$} & Crown slope & $\begin{array}{l}\text { minimum, maximum, range, mean, standard } \\
\text { deviation, sum, median, coefficient of } \\
\text { variation median, area }\end{array}$ & $\begin{array}{l}\text { (Original to this } \\
\text { research) }\end{array}$ \\
\hline & $\begin{array}{l}\text { Skewness of crown height } \\
\text { values }\end{array}$ & Skewness & Donoghue et al. 2007 \\
\hline & Crown Area & Area of polygon & Kin et al. 2011 \\
\hline & $\begin{array}{l}\text { Distribution of height points } \\
\text { (first returns) }\end{array}$ & $\begin{array}{l}\text { Variance, sum, standard deviation, } \\
\text { maximum, minimum, average, proportion }\end{array}$ & Briggs et al. 2011 \\
\hline \multirow[t]{4}{*}{$\begin{array}{l}\text { Internal } \\
\text { Attributes }\end{array}$} & $\begin{array}{l}\text { Proportion of returns per } \\
\text { quartile and } 90^{\text {th }} \%\end{array}$ & $\begin{array}{l}\text { Proportion of total at } 25,50,75 \text { and } 90^{\text {th }} \\
\text { percentiles }\end{array}$ & Donoghue et al. 2007 \\
\hline & $\begin{array}{l}\text { Proportion of first returns as } \\
\text { a proportion of to all returns }\end{array}$ & Proportion & Donoghue et al. 2007 \\
\hline & $\begin{array}{l}\text { Proportion of last returns as } \\
\text { a proportion of all returns }\end{array}$ & Proportion & Donoghue et al. 2007 \\
\hline & $\begin{array}{l}\text { Proportion of intermediate } \\
\text { returns as a proportion of all } \\
\text { returns }\end{array}$ & Proportion & Donoghue et al. 2007 \\
\hline
\end{tabular}

Most of the attributes could not directly be extracted using existing tools in ArcGIS. With attributes such as skewness of first return height, I had to create my own Python scripts to aid in the attribute extraction. For instance, the median value of the CHM was calculated by creating a script which multiplied the raster dataset by 100 , converted the values to integer format (required by the zonal command for the median calculation) conducted the zonal 
statistics procedure, and then finally divided the results by 100 to get the median as a real number.

The skewness of height values was calculated using a series of steps within ArcGIS (Appendix 2). Several spatial joins were conducted so that values such as the standard deviation of height and individual height values could be generated and used in the following equation:

$$
S=\frac{n}{(n-1)(n-2)} \sum\left(\frac{X_{\mathrm{i}}-\bar{X}}{\sigma}\right)^{3}
$$

Where, $S=$ skewness, $n=$ population size, $X_{\mathrm{i}}=$ individual height measurements, $i=$ measurement number, $\bar{X}=$ average height, $\boldsymbol{\sigma}=$ standard deviation.

The coefficient of variation (CV) was calculated as:

$$
c v=\frac{\sigma}{\bar{X}}
$$

Point counts and values derived from individual LiDAR points were captured using a spatial join. A spatial join assigns points that fall within a polygon to that polygon, thus allowing counts, mean values, minimum, maximum, standard deviation (STD) and variance values to be generated. Similarly, the counts for first returns, ground returns, intermediate returns and all returns were summed for each tree using a spatial join function in ArcGIS. Skewness, the CV and the percent of returns per vertical quartile were also calculated using a spatial join.

All of the attributes were assigned to each polygon assumed to represent a tree crown. The polygon datasets were then converted into raster datasets where every attribute was extracted to its own file, and each pixel within a polygon assigned the value of the attribute for that 
polygon. These files were then combined in a multi-layer, thematic file, analogous to a multiband image. This multi-layer file was then used in the classification.

The SVM classification was carried out using ENVI software. The SVM settings were chosen by adjusting the values until what appeared to be the best results were obtained. The final values I chose were radial basis function as the kernel type, the gamma in the kernel set to the inverse of the number of bands (0.021), the penalty parameter set at 550 , and the classification probability threshold set at 0 so that the entire image was classified.

For the NN classification also carried out of ENVI, I similarly used an empirical approach to set the parameters. The settings which appeared to produce the best results were a training threshold contribution of 0.9 , a logistic activation, a training rate of 0.1 , a training momentum of 0.9 , a training RMS exit criterion of $0.1,1$ hidden layer, 100,000 maximum training iterations, and a minimum output activation threshold of 0 .

For the CART I used a program called C-5 (also written as see-5) (Rulequest, Australia). I set the CART to create 50 iterations of the decision tree. The decision tree in this set of 50 decision trees which had the greatest fit to my data was used for the classification. These decision trees were converted into a Python script that I ran on the entire dataset to classify the segments.

Finally, an accuracy assessment was carried out on the segmentations and the classifications to judge the effectiveness of each method. 


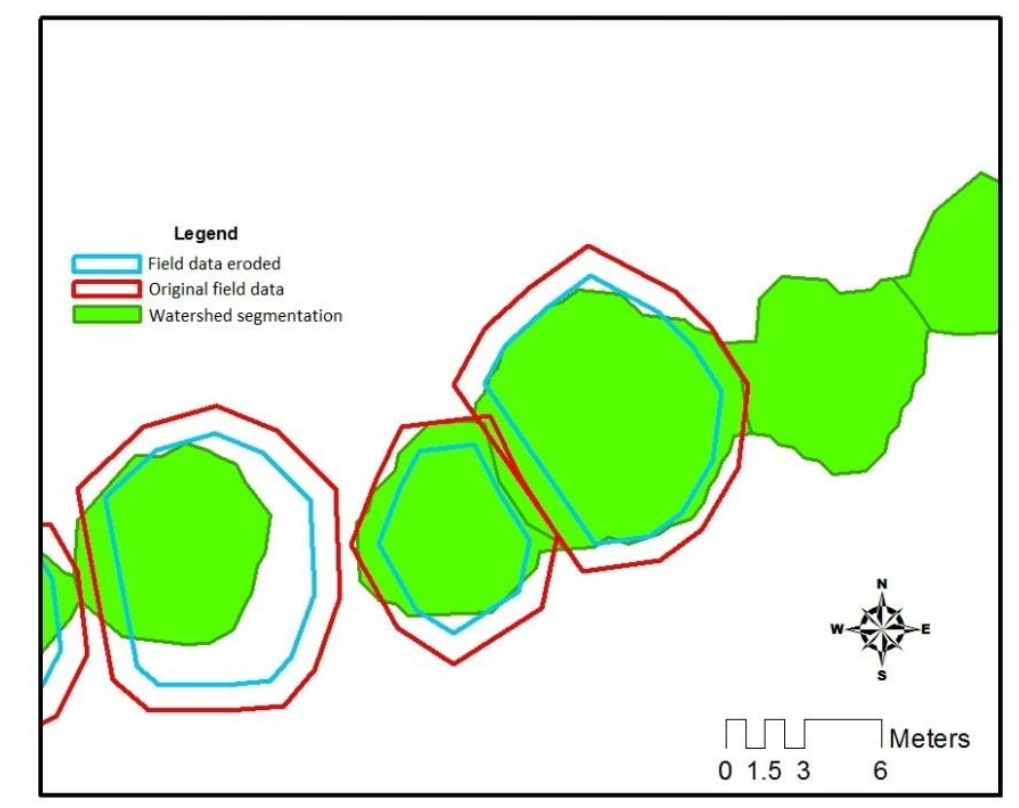

Figure 10: Comparison of the original and eroded field data, underlain by the watershed segmentation

The segmentation accuracy assessment was based on a comparison with the field measured crowns, and employed a fuzzy measure developed by Brandtberg et al. (2003) and implemented in a custom Imagine model (Appendix 3). The field raster polygons were first eroded by 1 pixel $(0.25 \mathrm{~m})$ so that adjacent trees were isolated (Figure 10). The 3-4 distance transformation, which assigns each pixel within a polygon with a value based on its distance from the crown edge, was applied to the field data. The method of assigning values to each pixel is a simplified Pythagorean theorem, where the hypotenuse of a triangle which is created by measuring the distance between two pixels is a simplified 3-4 right triangle (Borgefors, 1986). The aim of the transform is to weight centers of each crown with higher values than the edge pixels, since segmentation should be more accurate at finding the core of a tree, and is likely to be less accurate at locating the tree edges, which tend to be poorly defined. The 
maximum distance transform values are normalized to a range of $0-1$ for each tree regardless of size. The distance transform was then applied to the LiDAR CHM segmentation. The two distance transforms (field data and CHM segmentation) were then overlaid, and a measurement of segmentation agreement calculated for the entire scene (overall segmentation accuracy, A). If the two segmentations are similar, then the calculated value will be close to 1. Conversely the closer the value is to 0 , the more dissimilar the segmentation and the field data. Two additional metrics were calculated, omission error (OmErr), and commission error (ComErr). The measures of omission and commission were modified from Brandtberg et al.'s approach (2003). Normalization by the number of total pixels in each scene was necessary because the original measure was scene-specific and measured the total number of pixels of the particular error class. Omission error is a global measure of the trees present in the field data which were missed by the segmentation, (i.e. under segmentation). The omission error is a sum of the number of pixels that are non-zero in the distance transformation of the segmentation image normalized by the total number of tree pixels in the field data image. A high omission error indicates that the segmentation produced tree crown segments which were too small, under-representing tree crowns. Commission error is a global measure of image segmentations which do not exist in the field data image, (i.e. over-segmentation). The commission error is the sum of the number of pixels that are zero in the distance transformation of the field data image and non-zero in the segmentation image normalized by the total number of tree pixels in the segmentation image. A high commission error indicates that the CHM segmentation produced too many tree crowns, or crowns that extend outside the mapped boundaries of the crowns of the field data (Brandtberg et al. 2003). 
To evaluate the accuracy of each tree species classification, I trained the classifier using an $80 \%$ random selection of the field data by species, saving $20 \%$ for testing data. Prior to the classification I randomly divided all the field data into five groups, each comprising $20 \%$ of the data, which were used for testing the accuracy of the classification. The remaining $80 \%$ for each of the five replicates were used for training the classifier. The average value of all 5 classifications was used as an overall measure of classification accuracy (Figure 11). This allowed me to use all the field data as training as well as test data, increasing the precision of my classification accuracy (Czaplewski, and Stehman, 1998). I generalized from the pixel data to polygons by applying and classifying each polygon based on the majority class within that polygon. Polygons were then used for the confusion matrix calculation. Considering that tree identification is likely to be affected by segmentation accuracy, I also quantified the number of correctly classified trees based only upon those trees which were correctly segmented. A tree was regarded as correctly segmented if the CHM segment covered at least 50 percent of the pixels within a single tree crown polygon. Because the determination of correctly segmented trees is made separately in each segmentation, the number of trees used varies by segmentation method. I combined the August and the July results from the 2010 primary dataset for the classification accuracy assessment so that I could increase my sample size for the accuracy assessment. The sample size of the supplemental July 2008 data is too small to show the confusion matrices. 


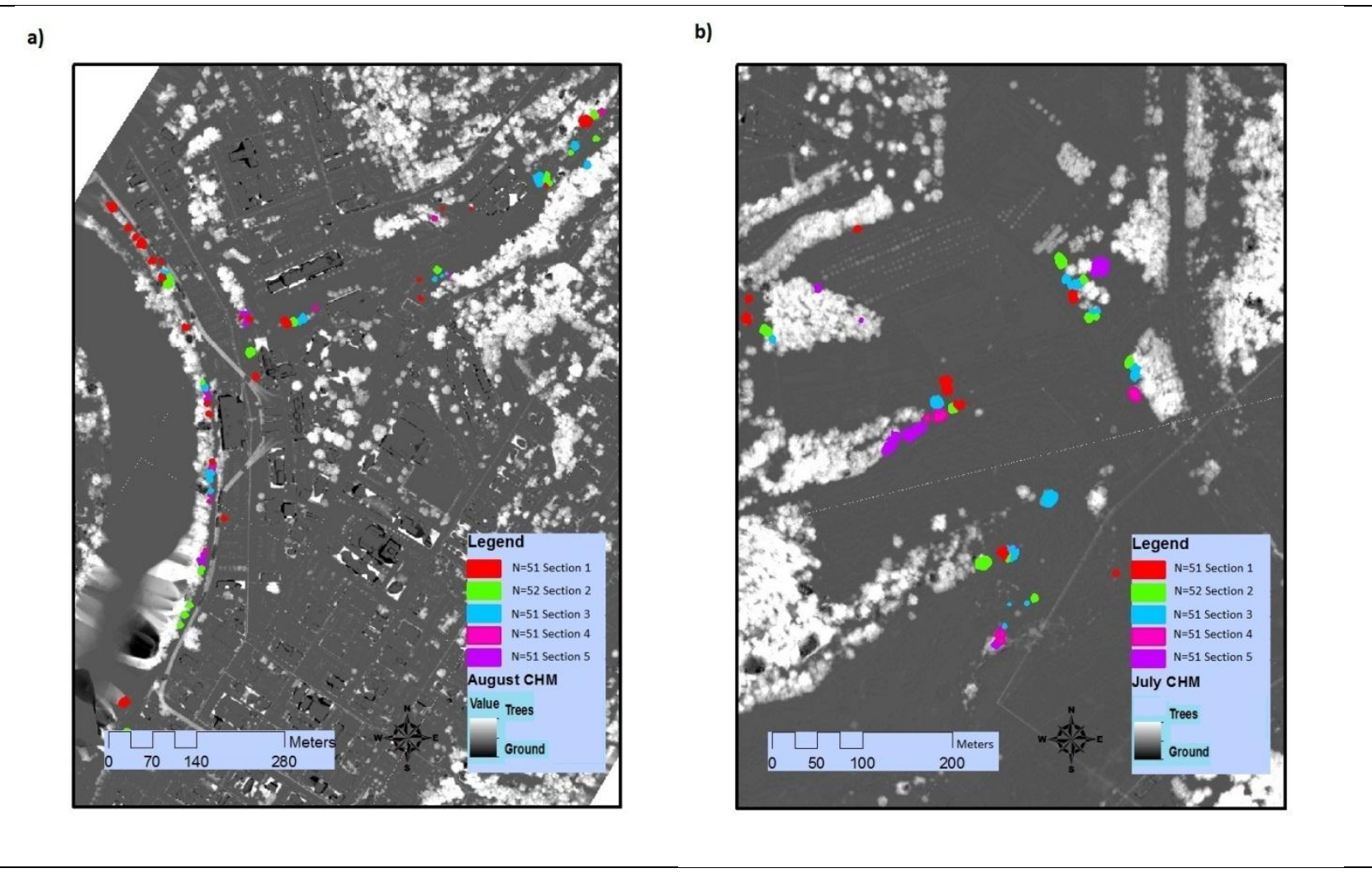

Figure 11: The division of the field data into 5 different test sets. (a) Monongahela River and Falling Run area. (b) WVU Organic Farm area 


\section{CHAPTER FOUR}

\section{$\underline{\text { RESULTS }}$}

\section{$\underline{\text { Tree Crown Segmentations }}$}

The tree crowns were most accurately separated using the watershed segmentation method, as indicated by the higher overall segmentation accuracy values for the watershed segmentation compared to the TreeVaW segmentation (Table 3, Figure 12). The overall segmentation accuracy value of the watershed segmentation is similar to the results received by Brandtberg et al. (2003), who obtained values that ranged from $0.21-0.35$.

\begin{tabular}{|l|l|c|c|c|}
\hline \multicolumn{3}{|c|}{ Table 3: The accuracy of the TreeVaW and watershed segmentations for the primary and Supplemental } \\
datasets. & \multicolumn{1}{|c|}{$\begin{array}{c}\text { Overall } \\
\text { Segmentation } \\
\text { Method }\end{array}$} & $\begin{array}{c}\text { Omission } \\
\text { Error } \\
\text { Accuracy (A) }\end{array}$ & $\begin{array}{c}\text { Commission } \\
\text { (OmErr) }\end{array}$ & $\begin{array}{c}\text { Error } \\
\text { (ComErr) }\end{array}$ \\
\hline Watershed & Primary July 2010 dataset & 0.248 & 0.009 & 0.229 \\
\hline & Primary August 2010 dataset & 0.238 & 0.011 & 0.237 \\
\hline & Supplemental July 2008 dataset & 0.132 & 0.304 & 0.568 \\
\hline TreeVaW & Primary July 2010 dataset & 0.172 & 0.591 & 0.141 \\
\hline & Primary August 2010 dataset & 0.192 & 0.483 & 0.134 \\
\hline & Supplemental July 2008 dataset & 0.085 & 0.944 & 0.205 \\
\hline
\end{tabular}



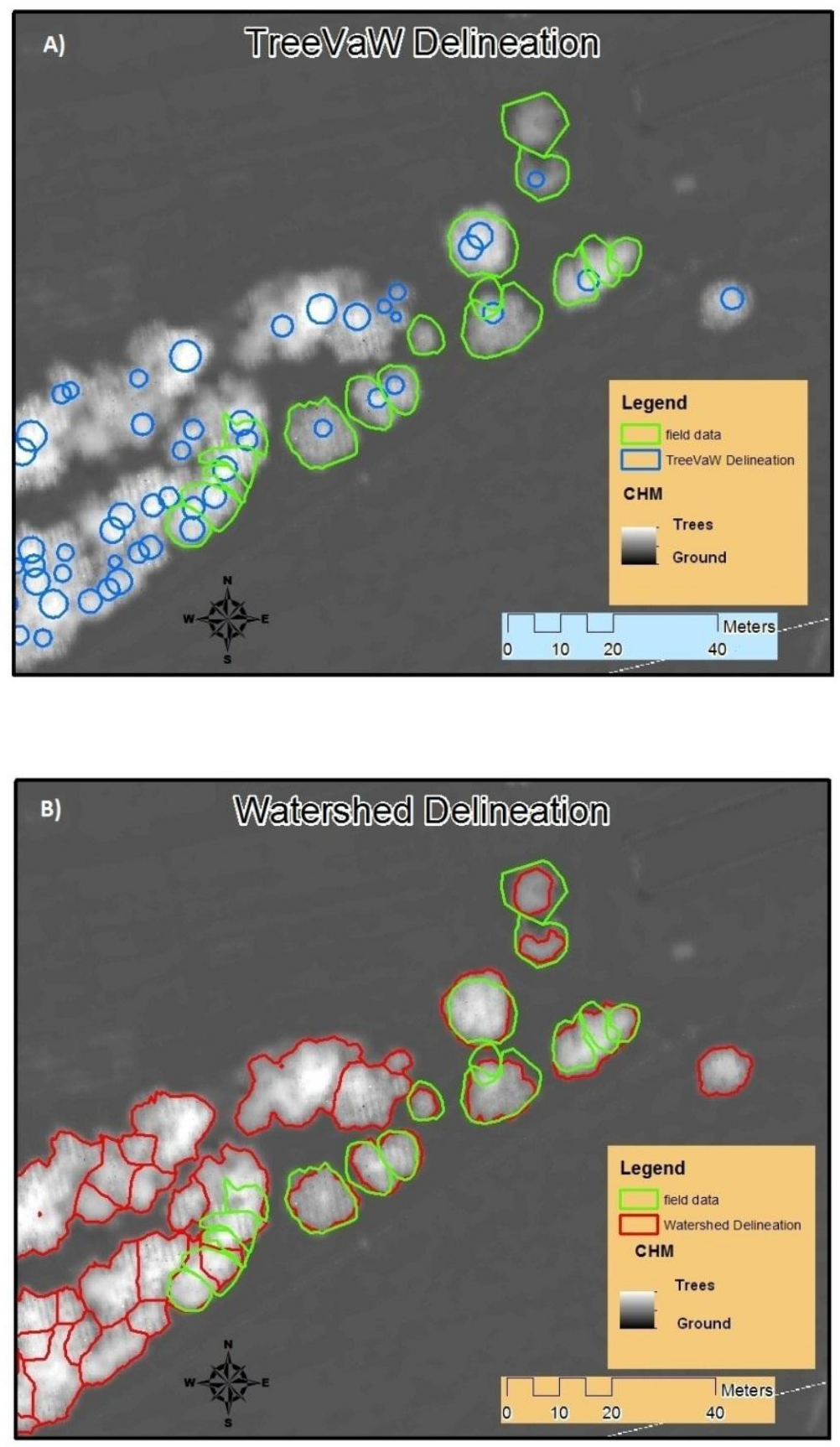

Figure 12: A comparison of the segmentation with the field data (a) TreeVaW segmentations (b) watershed segmentation 
The most striking feature of Table 3 is that TreeVaW had low commission error and higher omission error, whereas the watershed segmentation produced the opposite. Omission errors were notably higher for the TreeVaW segmentation, indicating that tree canopy boundaries as mapped by the program were in general too small to represent the tree crowns accurately.

Thus, much of the tree canopy was not mapped as part of any specific tree (Table 3, Figure 12 (a)). Commission errors were actually higher in the watershed segmentation than the TreeVaW segmentation (Table 3), which indicates that the watershed segments are in general undersegmented the canopy, tending towards an agglomeration of tree crowns.

The segmentation of the supplemental July 2008 dataset was less accurate than the primary 2010 datasets, producing lower overall segmentation accuracies and higher commission errors compared to the primary dataset (Table 3, Figure $12(b))$. This is reflected in the omission errors, which were very high for the TreeVaW segmentation, and the commission errors which were very high for the watershed segmentation, compared to the primary dataset.

Table 4: Percentages of each tree species which were segmented correctly for July and August 2010, and July 2008, based on a sample of 78 Ailanthus, 64 Other Deciduous, and 33 Coniferous trees, respectively.

\begin{tabular}{|l|c|c|c|c|}
\hline & \multicolumn{4}{|c|}{ Segmented Correctly (\%) } \\
\hline & $\begin{array}{c}\text { 2010 Watershed } \\
\text { Segmentation }\end{array}$ & $\begin{array}{c}\text { 2010 TreeVaW } \\
\text { Segmentation }\end{array}$ & $\begin{array}{c}\text { 2008- } \\
\text { Watershed } \\
\text { Segmentation }\end{array}$ & $\begin{array}{c}\text { 2008 - TreeVaW } \\
\text { Segmentation }\end{array}$ \\
\hline Ailanthus & 67 & 30 & 15 & 13 \\
\hline Other Deciduous & 97 & 64 & 8 & 16 \\
\hline Coniferous & 97 & 68 & N/A & N/A \\
\hline
\end{tabular}


Table 4 shows the percentage of trees which were segmented correctly by the watershed and TreeVaW segmentations. There is a clear pattern in the primary and supplemental datasets in which $30 \%$ fewer Ailanthus altissima trees were segmented correctly with both the watershed segmentation and the TreeVaW segmentation than the coniferous and other deciduous trees. This may be a result of the way in which Ailanthus trees grow close together and form indistinguishable canopy groups, partly a result of the way they reproduce clonally from root and stump sprouts. 


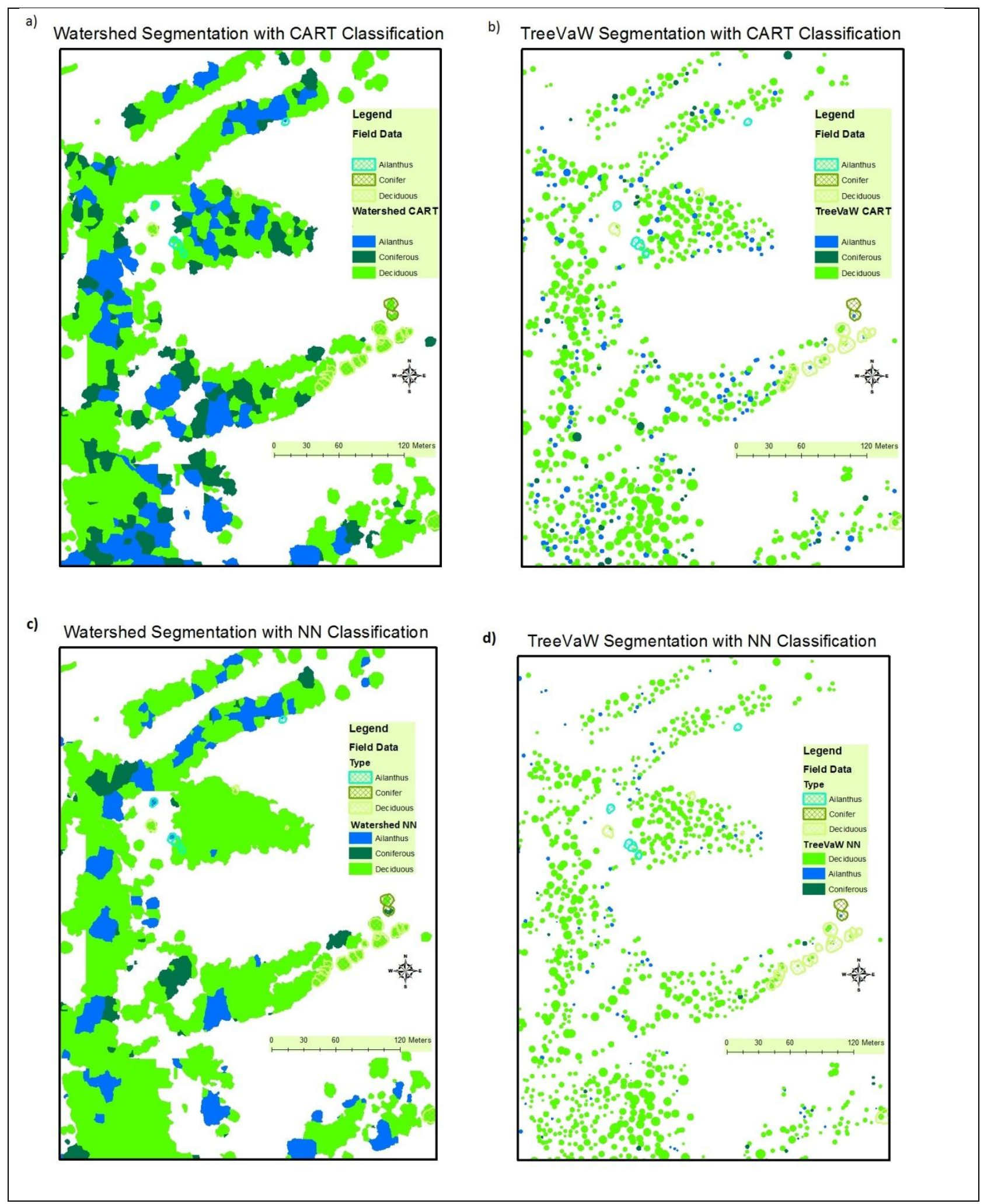


e) Watershed Segmentation with SVM Classification

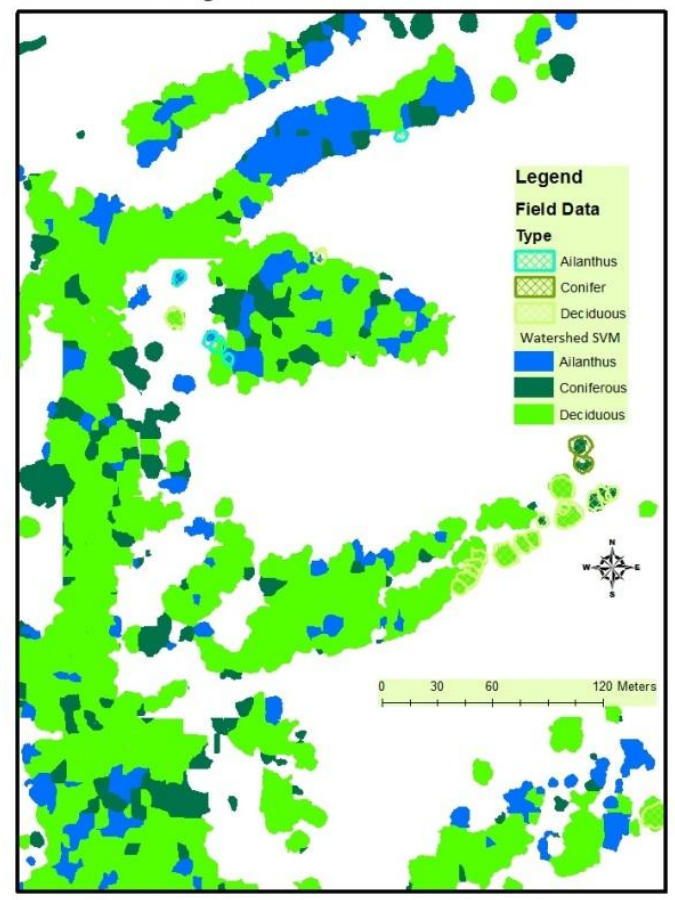

f)

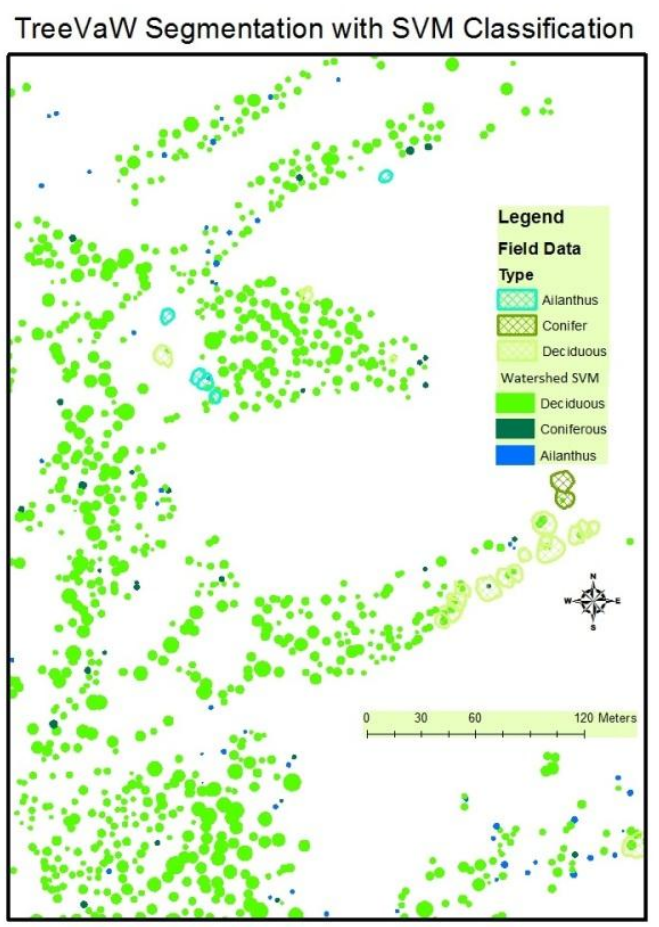

G)

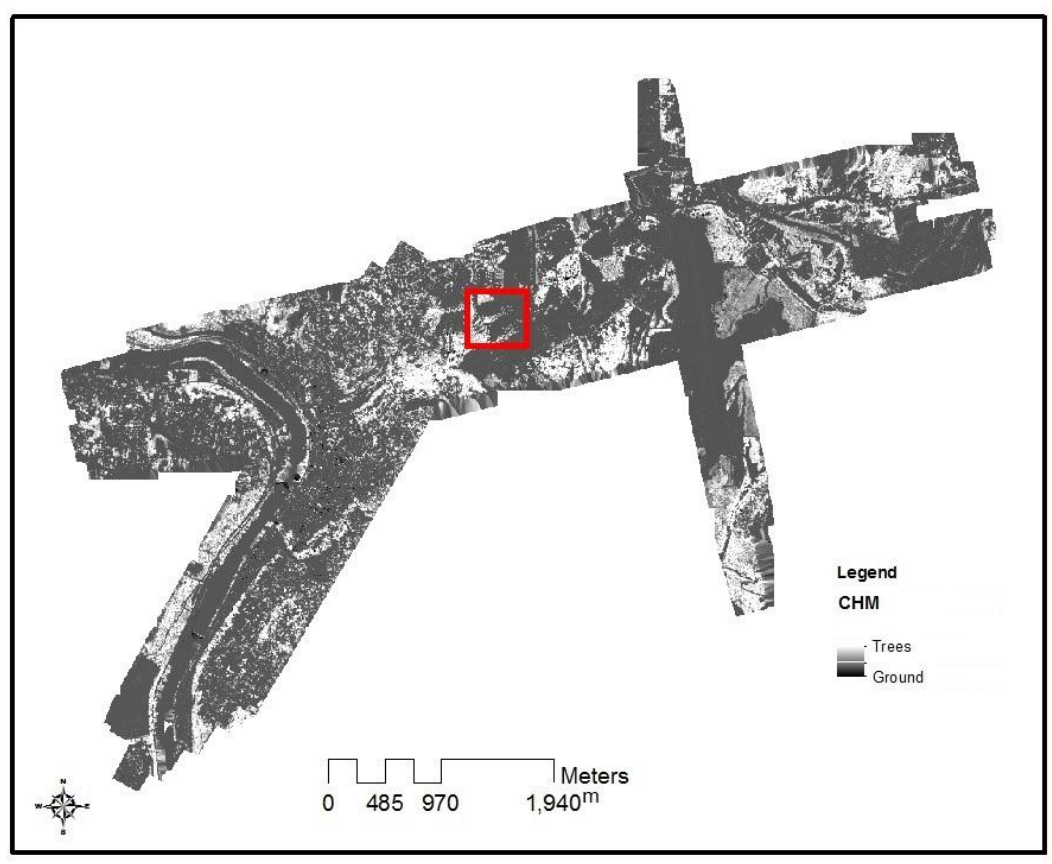

Figure 13: A comparison of the classifications of the primary dataset (July 2010). (a)Watershed segmentation with CART. (b) TreeVaW segmentation with CART. (c) Watershed segmentation with NN. (d) TreeVaW segmentation with NN. (e) Watershed segmentation with SVM. (f) TreeVaW segmentation with SVM. (g) The extent of images $a-f$ in the CHM. 


\section{Classifications}

\section{$\underline{C A R T}$}

Table 5: Error matrix for the CART classification of the watershed segmentation calculated using trees which were correctly segmented. $A=$ Ailanthus, $O D=$ Other deciduous, $C=$ Coniferous. Watershed segmentation (Values in the table represent number of trees $n=291,0.69$ overall accuracy).

\begin{tabular}{|c|c|c|c|c|c|}
\hline & & \multicolumn{3}{|c|}{ Classification } & \\
\hline & & A & OD & $\mathrm{C}$ & $\begin{array}{l}\text { Producers' } \\
\text { Accuracy }\end{array}$ \\
\hline & $\mathrm{A}$ & 51 & 28 & 6 & 0.60 \\
\hline \multirow{3}{*}{ 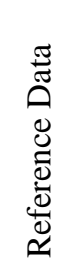 } & OD & 33 & 80 & 7 & 0.66 \\
\hline & $\mathrm{C}$ & 1 & 14 & 71 & 0.82 \\
\hline & Users' Accuracy & 0.60 & 0.66 & 0.85 & \\
\hline
\end{tabular}

Figure 13 (a) and (b) shows the CART classification applied to the watershed and TreeVaW segmentations. The CART classification users' accuracy was generally similar for the watershed and the TreeVaw segmentations, although overall classification accuracy and Ailanthus producers' accuracy were higher with the TreeVaW segmentation (Table 5). However, CART applied to the watershed segmentation classified the coniferous trees more accurately than with TreeVaW segmentation, as indicated by both the users' and producers' accuracy (Table 6). This conifer result is probably because the watershed segmentation works well with the clearly defined, conical shape of the conifers, and the conifers have a distinctive shape that CART is able to exploit. 
The CART classification of the TreeVaW segmentation was somewhat successful with the classification of the other deciduous trees, which had a high users' and moderate producers' accuracy (Table 6). However, Ailanthus trees and other deciduous trees were often confused, resulting in a users' accuracy much lower than that of the watershed segmentation.

Table 6: Error matrix for the CART classification of the TreeVaW segmentation calculated using trees which were correctly segmented. $\mathrm{A}=$ Ailanthus, $\mathrm{OD}=$ Other deciduous, $\mathrm{C}=$ Coniferous. TreeVaW segmentation (Values in the table represent number of trees $n=294$ and overall accuracy $=0.74$ )

\begin{tabular}{|c|c|c|c|c|c|}
\hline & & & $\mathrm{Cla}$ & & \\
\hline & & $\bar{A}$ & OD & $\mathrm{C}$ & $\begin{array}{l}\text { Producers' } \\
\text { Accuracy }\end{array}$ \\
\hline & A & 20 & 1 & 0 & 0.95 \\
\hline 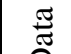 & OD & 20 & 75 & 8 & 0.72 \\
\hline$\stackrel{\circlearrowright}{0}$ & C & 1 & 16 & 39 & 0.69 \\
\hline$\frac{\tilde{\omega}}{\mathscr{L}}$ & Users' Accuracy & 0.48 & 0.81 & 0.82 & \\
\hline
\end{tabular}

$\underline{N N}$

The NN classification using the watershed segmentation (Table 7) produced a lower users' accuracy for the Ailanthus species than the CART classification applied to the watershed segmentation, as well as compared to the CART classification applied to the TreeVaW segmentation. The NN classification also resulted in a low producers' accuracy for Ailanthus, and the overall classification accuracy was low (0.54). 


\begin{tabular}{|c|c|c|c|c|c|}
\hline \multirow{6}{*}{ 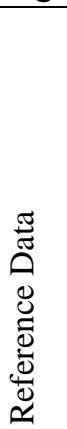 } & & \multicolumn{3}{|c|}{ Classification } & \multirow[b]{2}{*}{$\begin{array}{l}\text { Producers' } \\
\text { Accuracy }\end{array}$} \\
\hline & & $\mathrm{A}$ & OD & $\mathrm{C}$ & \\
\hline & $\mathrm{A}$ & 39 & 30 & 12 & 0.48 \\
\hline & OD & 33 & 78 & 28 & 0.56 \\
\hline & $\mathrm{C}$ & 20 & 14 & 44 & 0.56 \\
\hline & Users' Accuracy & 0.42 & 0.63 & 0.52 & \\
\hline
\end{tabular}

The NN classification coupled with the TreeVaW segmentation produced a higher overall accuracy than when applied to the watershed segmentation (Table 8). It also produced the highest users' accuracy for identifying Ailanthus trees of the entire study (0.76). However, the producers' accuracy was low (0.51).

Table 8: Error matrix for the NN classification of the TreeVaW segmentation calculated using trees which were correctly segmented. $\mathrm{A}=$ Ailanthus, $\mathrm{OD}=$ Other deciduous, $\mathrm{C}=$ Coniferous. TreeVaW segmentation (Values in the table represent number of trees $n=294$ and overall accuracy $=0.62$ )

\begin{tabular}{|c|c|c|c|c|c|}
\hline & & \multicolumn{3}{|c|}{ Classification } & \\
\hline & & $\mathrm{A}$ & OD & $\mathrm{C}$ & $\begin{array}{l}\text { Producers' } \\
\text { Accuracy }\end{array}$ \\
\hline & A & 31 & 25 & 5 & 0.51 \\
\hline \multirow{3}{*}{ 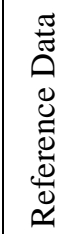 } & OD & 10 & 48 & 24 & 0.59 \\
\hline & $\mathrm{C}$ & 0 & 4 & 30 & 0.88 \\
\hline & Users' Accuracy & 0.76 & 0.62 & 0.51 & \\
\hline
\end{tabular}

$\underline{S V M}$

The SVM classification of the watershed segmentation produced moderately high Ailanthus producers' accuracy and moderately high users' accuracy for other deciduous and coniferous trees 
(Table 9). However, the overall accuracy of the SVM was below that of the CART, and only slightly above the NN watershed segmentation classification, but below the accuracy of the TreeVaW NN classification.

Table 9: Error matrix for the SVM classification of the watershed segmentation calculated using trees which were correctly segmented. $A=$ Ailanthus, $O D=$ Other deciduous, $C=$ Coniferous. Watershed segmentation (Values in the table represent number of trees $n=291,0.66$ overall accuracy)

\begin{tabular}{|c|c|c|c|c|c|}
\hline & & \multicolumn{3}{|c|}{ Classification } & \\
\hline & & A & OD & $\mathrm{C}$ & $\begin{array}{l}\text { Producers' } \\
\text { Accuracy }\end{array}$ \\
\hline & A & 54 & 23 & 2 & 0.68 \\
\hline \multirow{3}{*}{ 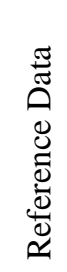 } & OD & 37 & 88 & 24 & 0.59 \\
\hline & $\mathrm{C}$ & 2 & 11 & 53 & 0.80 \\
\hline & Users' Accuracy & 0.58 & 0.72 & 0.67 & \\
\hline
\end{tabular}

The SVM classification of the TreeVaW segmentation handled the separation of the Ailanthus species better than CART classification of the TreeVaW segmentation, producing a users' accuracy of 0.58 , which was higher than the users' accuracy obtained by the watershed segmentation (0.48). On the other hand, the overall accuracy was relatively low (0.58) (Table 10). 


\begin{tabular}{|c|c|c|c|c|c|}
\hline \multirow{6}{*}{ 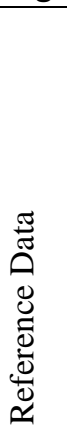 } & & \multicolumn{3}{|c|}{ Classification } & \multirow[b]{2}{*}{$\begin{array}{l}\text { Producers' } \\
\text { Accuracy }\end{array}$} \\
\hline & & A & OD & $\mathrm{C}$ & \\
\hline & $\mathrm{A}$ & 24 & 29 & 7 & 0.40 \\
\hline & OD & 15 & 47 & 15 & 0.61 \\
\hline & $\mathrm{C}$ & 2 & 3 & 31 & 0.86 \\
\hline & Users' Accuracy & 0.58 & 0.59 & 0.58 & \\
\hline
\end{tabular}

\section{Summary of Classification Using Correctly Segmented Trees}

In summary, the CART classification usually has the highest overall accuracy, regardless of segmentation method (Table 11). However, the users' accuracy for the Ailanthus does not necessarily follow the overall accuracy pattern, and is also not consistent between the primary and supplemental datasets. This suggests that the attributes which I chose were handled differently by each classification method, and that segmentation accuracy is not the only factor is classification accuracy.

\begin{tabular}{|c|c|c|c|c|c|c|c|c|c|c|c|c|}
\hline \multicolumn{1}{|c|}{ Table 11: Summary of the overall users' accuracy as the percentage of the classification based } \\
all trees in the field data \\
\hline
\end{tabular}


The calculation of classification accuracy is based on the total number of trees in the field dataset includes trees that were not correctly segmented, and therefore are automatically counted as errors (Table 12). This allows for the combined effectiveness of the segmentation and classification methods to be compared. I have chosen to focus on users' accuracy to summarize the classification accuracies, because the users' accuracy represents the probability that a tree will appear on the ground as it has been classed in the classification (Congalton 1991). The accuracy of the tree classification when comparing only the trees which were correctly segmented was much higher than the measure using all the field data, especially for the TreeVaW segmentation (Table 12).

Table 12: Summary of overall users' accuracy as the percentage of the classifications based upon trees which were correctly segmented

\begin{tabular}{|l|c|c|c|c|c|c|c|c|c|c|c|c|}
\hline & \multicolumn{3}{|c|}{$\begin{array}{c}\text { Primary Data - } \\
\text { Watershed }\end{array}$} & \multicolumn{3}{|c|}{ Primary Data -TreeVaW } & \multicolumn{2}{|c|}{$\begin{array}{c}\text { Supplemental Data - } \\
\text { Watershed }\end{array}$} & \multicolumn{3}{|c|}{$\begin{array}{c}\text { Supplemental Data - } \\
\text { TreeVaW }\end{array}$} \\
\cline { 2 - 14 } & CART & NN & SVM & CART & NN & SVM & CART & NN & SVM & CART & NN & SVM \\
\hline Ailanthus & 60 & 42 & 58 & 49 & 76 & 59 & 89 & 37 & 93 & 87 & 10 & 61 \\
\hline $\begin{array}{l}\text { Other } \\
\text { Deciduous }\end{array}$ & 66 & 64 & 72 & 82 & 62 & 59 & 79 & 82 & 85 & 71 & 96 & 70 \\
\hline Coniferous & 85 & 52 & 67 & 83 & 51 & 58 & N/A & N/A & N/A & N/A & N/A & N/A \\
\hline Overall & 69 & 53 & 66 & 74 & 63 & 58 & 84 & 59 & 89 & 79 & 53 & 65 \\
\hline
\end{tabular}

The overall accuracies of the classifications show that the watershed segmentation with the CART classification method produces the best overall result in both the preliminary and 
supplemental datasets, followed by the watershed segmentation with SVM classification (Table 11). However, the watershed segmentation with the SVM classification is actually more accurate at classifying the Ailanthus species than the CART or the NN classification.

Looking specifically at the TreeVaW segmentation applied to the primary dataset, on the other hand, CART is the most successful classifier overall, but the least successful at classifying Ailanthus, where both NN and SVM out-compete it. In contrast, the users' accuracy of Ailanthus classification is relatively high for the CART classification applied to the supplemental dataset. The method with the lowest overall accuracy with the TreeVaW segmentation was the SVM of the preliminary dataset.

The overall classification accuracy of the supplemental July 2008 dataset is relatively low compared to the primary dataset, probably because the LiDAR data for the supplemental dataset has a lower point-density than the primary LiDAR dataset, and therefore produced a poor segmentation (Tables 3 and 4). The classification results of the supplemental dataset do however in general mirror the primary dataset, in that SVM classifications of the watershed segmentation produced the best Ailanthus classification, and NN the worst. CART is the best overall classifier. The classification of the TreeVaW segmentation of supplemental dataset also mirrors that of the primary dataset, with the NN classification of the TreeVaW segmentation resulting in a lower users' accuracy (20.6\%) than was derived from the NN classification of the watershed segmentation (24.4\%).

\section{Important Attributes}




\begin{tabular}{|l|c|l|c|}
\hline \multicolumn{4}{|l|}{ Table 13: Attributes ranked by number of appearances in the CART decision trees. } \\
\hline \multicolumn{1}{|c|}{ Most commonly used attributes } & Rank & \multicolumn{1}{c|}{ Least used attributes } & Rank \\
\hline Standard Deviation of Crown Slope & 1 & Mean Height Value & 55 \\
\hline Percentage of points in Quartile 2 & 2 & Range of Elevations & 54 \\
\hline Percentage of first returns & 3 & Range of Slope & 53 \\
\hline Percentage of interior points & 4 & CV of Slope & 52 \\
\hline STD of Elevation & 5 & Sum of Intensity values & 51 \\
\hline Maximum Elevation & 6 & Variability of the maximum Z value & 50 \\
\hline Percentage of ground returns & 7 & Sum of Z maximum values & 49 \\
\hline Maximum intensity & 8 & Sum of Elevation Values & 48 \\
\hline Percentage of points in Quartile3 & 9 & Maximum Height Values & 47 \\
\hline Percentage of points in Quartile1 & 10 & Area of Crown & 46 \\
\hline STD Intensity & 11 & CV of Intensity & 45 \\
\hline Median Intensity & 12 & Sum of Slope & 44 \\
\hline Mean Slope & 13 & Minimum Slope & 43 \\
\hline Percentage of points in $90^{\text {th }}$ percentile & 14 & STD of Z Max Values & 42 \\
\hline CV of Elevation & 15 & STD of Height & 41 \\
\hline
\end{tabular}

Using all of the decision trees from the CART analysis, I created a ranking of the most useful and least useful attributes from the data (Table 13), based on how often they appeared in the decision tree. The most useful attribute was standard deviation (STD) of crown slope, which is an attribute developed in this study. Some of the other very useful attributes included the vertical position of points within the tree (percentage of first returns, percentage of ground returns, percentage of interior points, percentage of points in quartile 1 (Q1) and percentage of points in quartile $3(\mathrm{Q} 3)$ ), which is evidence that the different tree species produce different tree profiles because of their shape, density of interior leaves and twigs, and the leaf and twig density at different heights of the tree. It is notable that ground elevation values were such a common factor in species identification (CV elevation, STD of elevation, maximum elevation). This suggests that there was an elevational association with tree species. However, this may be a spurious association due in a small sample size and the clumped nature of the different trees 
in the training data. Finally, intensity seemed to be a common factor in species identification (maximum intensity, STD of intensity, mean intensity), which provides evidence that raw, nonnormalized LiDAR data can still use intensity values as species indicators. This idea supports the findings of Orka et al. (2008) who also used raw intensity values to classify species.

The least used attributes mostly had to do with crown slope, tree height and area. Tree height and canopy size likely vary greatly for trees that are relatively young, which is common in urban environments. Crown area was not used much, which was probably due to problems with the segmentation method. Finally, I believe that the several slope values which were rarely used (range of slope, sum of slope values, minimum slope, CV of slope), were not chosen frequently in the CART classification because the STD of crown slope provided such an effective indicator of the overall tree crown shape that the other crown slope measures were redundant.

This supposition is supported by the fact that when the standard deviation of slope is eliminated from the CART decision tree, it is replaced by the values of mean slope and CV of slope, which then become the $3^{\text {rd }}$ and $5^{\text {th }}$ most used attributes. The accuracy of the CART classification without the attribute of STD of slope went down $6 \%$ for Ailanthus, and $11 \%$ overall.

One potential criticism of Table 13 is that it does not differentiate between nodes at the top of the decision tree that may affect many samples in the classification, and nodes at near the bottom of the decision tree that may affect very few samples. To address this concern, I then created a table ranking the attributes based on the number of trees that each attribute classified (Table 14). This new table has some important differences in comparison to the 
original, unweighted data (Table 13). In particular, Table 14 includes as one of the most important attributes mean intensity, which is commonly identified as one of the most effective attributes in other studies (Kin et al. 2011). Crown area, which is also usually included as one of the most effective attributes, but was ranked amongst the least used attributes in Table 13, was ranked as of intermediate importance, and is therefore not listed in Table 14. One important similarity between Tables 13 and 14 is that STD of slope is ranked as the most effective attribute for both methods of ranking attributes.

\begin{tabular}{|c|c|c|c|}
\hline Most commonly used attributes & Rank & Least used attributes & Rank \\
\hline Standard Deviation of Crown Slope & 1 & Sum of Intensity & 55 \\
\hline Percentage of first returns & 2 & Intensity Coefficient of Variation & 54 \\
\hline Percentage of Internal Points & 3 & Elevation of tree in the $90^{\text {th }}$ percentile & 53 \\
\hline Maximum Intensity & 4 & Elevation Range & 52 \\
\hline STD of Elevation & 5 & Height of tree in the $90^{\text {th }}$ percentile & 51 \\
\hline Percentage of points in Quartile 2 & 6 & Mean Height & 50 \\
\hline Percentage of points in Quartile 1 & 7 & Height Coefficient of Variation & 49 \\
\hline Maximum Elevation & 8 & STD of maximum $Z$ value & 48 \\
\hline Percentage of points in Quartile 4 & 9 & Slope Range & 47 \\
\hline Mean Intensity & 10 & Maximum Height & 46 \\
\hline Tree height at Quartile 2 & 11 & Sum of Elevation values & 45 \\
\hline Minimum Elevation & 12 & Average Z Max value & 44 \\
\hline Mean Elevation & 13 & Median Elevation & 43 \\
\hline STD Intensity & 14 & Height Range & 42 \\
\hline CV of Elevation & 15 & Maximum Slope & 41 \\
\hline
\end{tabular}

Finally, to visualize the distribution of the attributes used, I graphed the four most useful attributes as a function of species (standard deviation of crown slope, percentage of first returns, percentage of interior returns, maximum intensity). It is important to recognize that univariate plots cannot display covariance between classes that may be important in classification, and therefore an overlap between classes in a univariate plot does not 
necessarily mean the variable is not useful. Nevertheless, univariate plots are effective for showing distributions of variables.

Figure 14, the box plot for the standard deviation of slope, shows that Ailanthus altissima distribution is somewhat similar to that of other deciduous trees, and a little higher than the coniferous trees. The median value of Ailanthus (14.3) is between other deciduous (13.3) and coniferous trees (15.1).

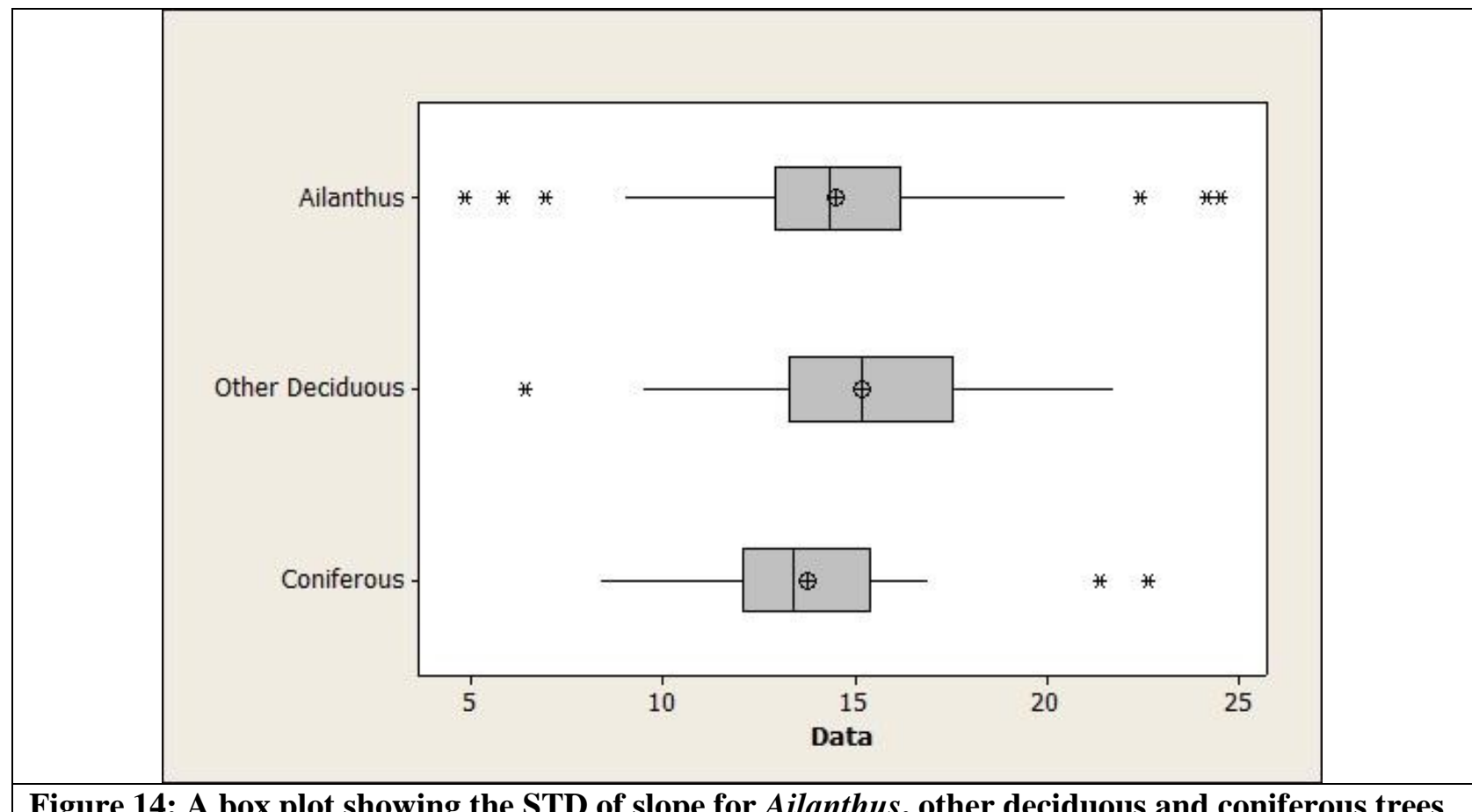

Figure 14: A box plot showing the STD of slope for Ailanthus, other deciduous and coniferous trees 
The percentage of first returns for the Ailanthus species is generally higher than both the deciduous and coniferous trees (Figure 15). The mean value for Ailanthus (64.9) was higher than both the other deciduous trees (48.7) and coniferous trees (51.5). The median values were also higher for Ailanthus (60.6), than other deciduous (43.7) and coniferous trees (53.4).

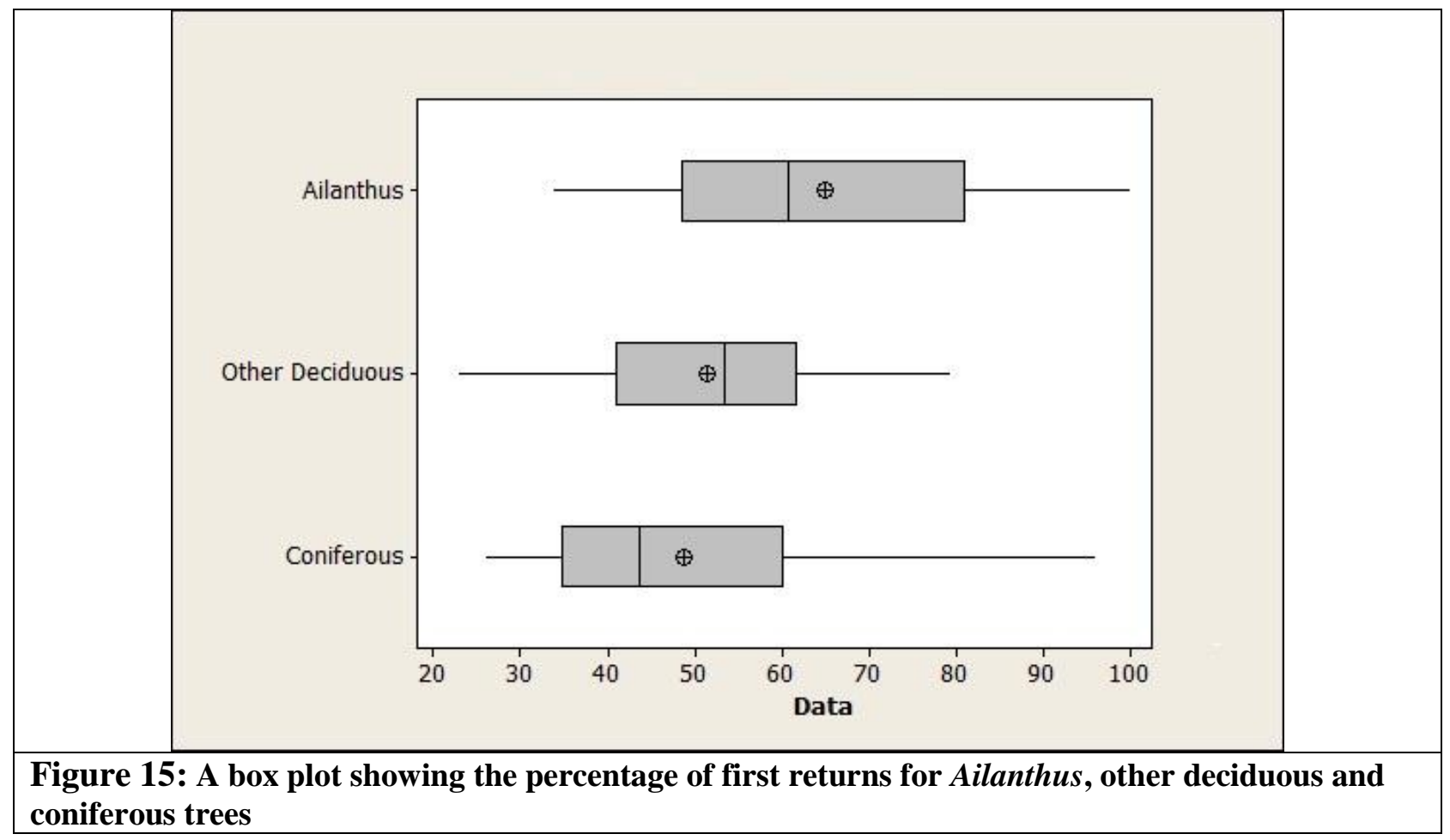


The percentage of intermediate returns value is generally lower for Ailanthus species

(Figure 16). The mean value for Ailanthus (25.6) is lower than both coniferous (40.5) and deciduous (35.1) trees. The median value for Ailanthus (29.1) is lower than other deciduous (47.5) and coniferous trees (33.5).

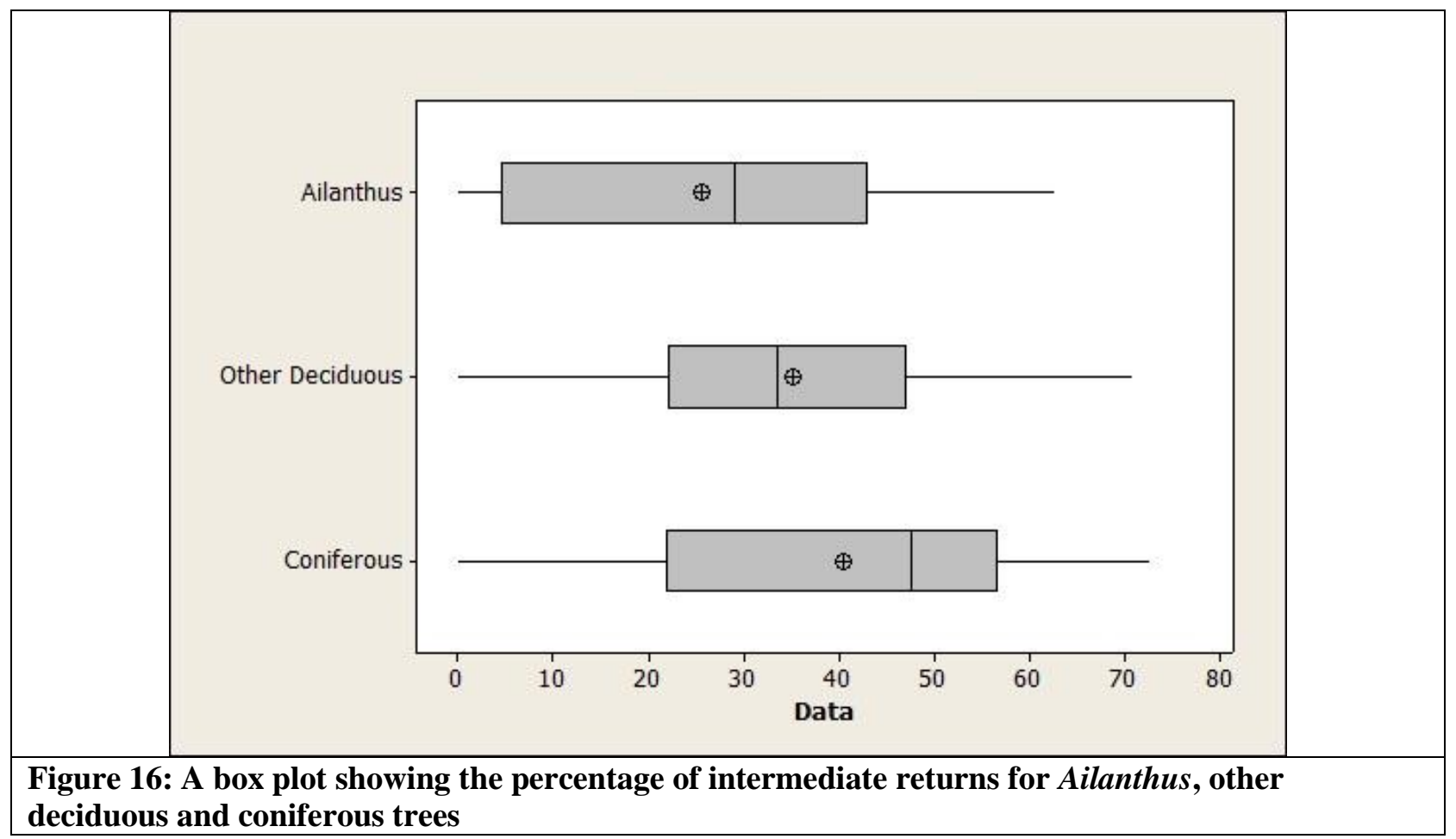

The maximum intensity values for Ailanthus are higher than the values of other deciduous and coniferous trees (Figure 17). The mean value of the maximum intensity for the Ailanthus tree (176.3) is higher than other deciduous trees (167.4), and coniferous trees (157.5). Similarly, the median for Ailanthus (173.7) is higher than both other deciduous (165.6) and coniferous trees (162.7). 


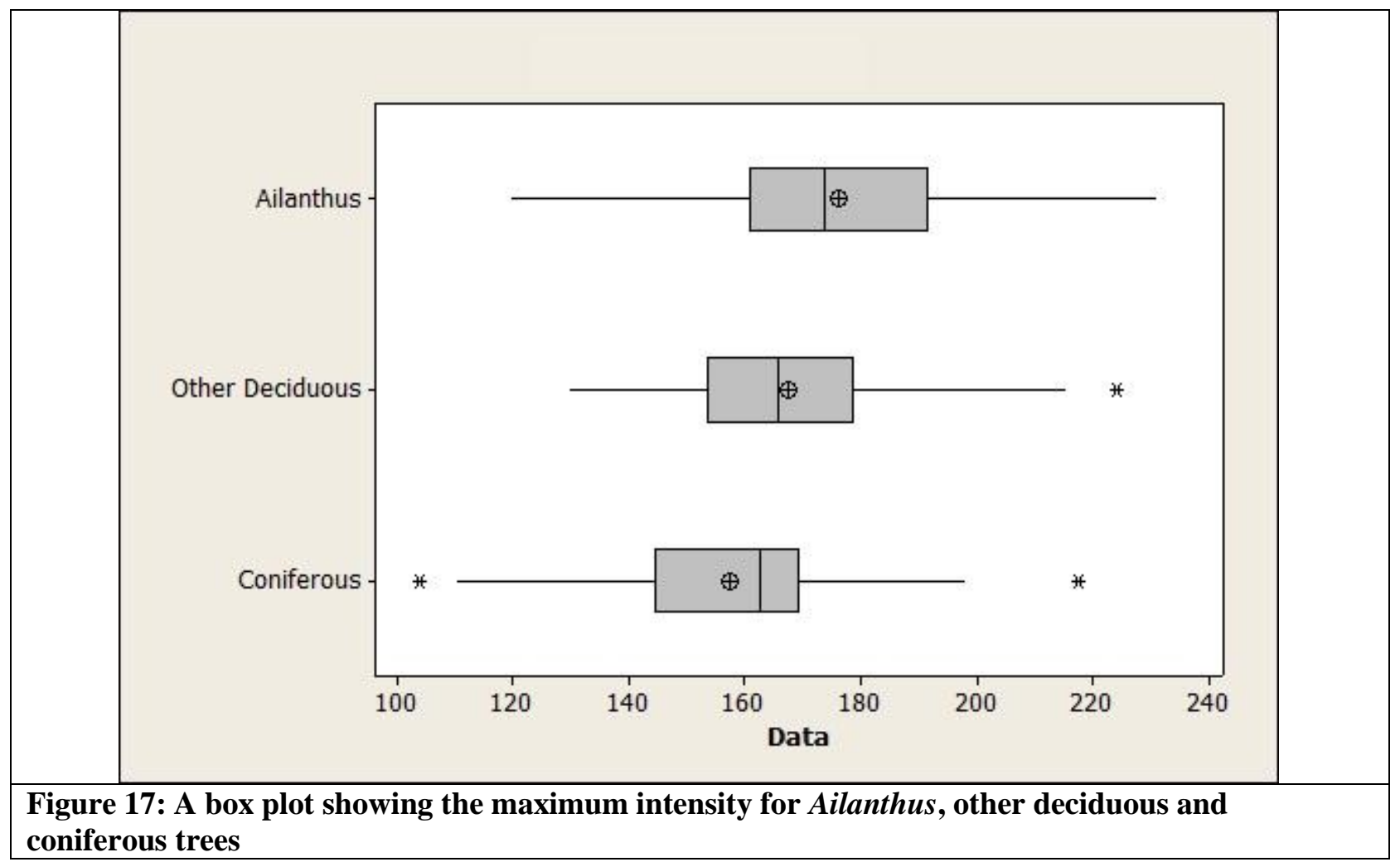

In summary, the STD of crown slope attribute shows high degree of overlap, suggesting that its importance is due to interactions with other attributes. The percentage of first and intermediate returns suggests that Ailanthus canopy is relatively opaque, and most returns are from the outer surface of the canopy, whereas other deciduous and coniferous trees apparently allow greater penetration. The higher maximum intensity of Ailanthus may be a consequence of this greater opacity, and may also relate to higher reflectance of the Ailanthus leaves. 


\section{CHAPTER FIVE}

\section{$\underline{\text { DISCUSSION }}$}

\section{Preprocessing Methods}

$\underline{\text { CHM }}$

The CHM was generated from an IDW interpolation with $0.25 \mathrm{~m}$ pixels, and the parameters were especially adjusted to generate complete tree crowns by including a large number of neighbors in the IDW algorithm to eliminate striping in the tree crowns caused by the sweeping motion of the laser scanner which produced gaps between points. This made the tree crown segments less jagged, but also smoothed the forest canopies, and made them more difficult to separate. This suggests that alternative interpolation methods should be considered that will retain the shape of the tree crowns while eliminating the striping caused by the laser scanner.

Currently, there are filters which can be applied to the LiDAR data to extract features such as buildings, trees, and water, but they are not equally effective with every dataset, especially if the data is broken into sections in order to handle the large amounts of data. When the data is separated into multiple tiles, the filters do not work as well with the objects on the edges of the tile. These limitations in filtering lead to artifacts remaining in the $\mathrm{CHM}$, such as buildings, or sections of buildings, public transit systems, lampposts, telephone wires, cars, and airconditioning units, all of which may be segmented as trees and included in the classification (Figure 18). Ideally, therefore, an additional pre-processing step should be carried out to 
remove these artifacts. Shape as well as attributes of these artifacts, such as intensity and slope, differ from those of trees which would make them easy to identify.

The creation of the $\mathrm{CHM}$ is extremely important, and errors in the $\mathrm{CHM}$ filter will necessarily produce an inaccurate segmentation, and decrease the accuracy of the subsequent classification. 


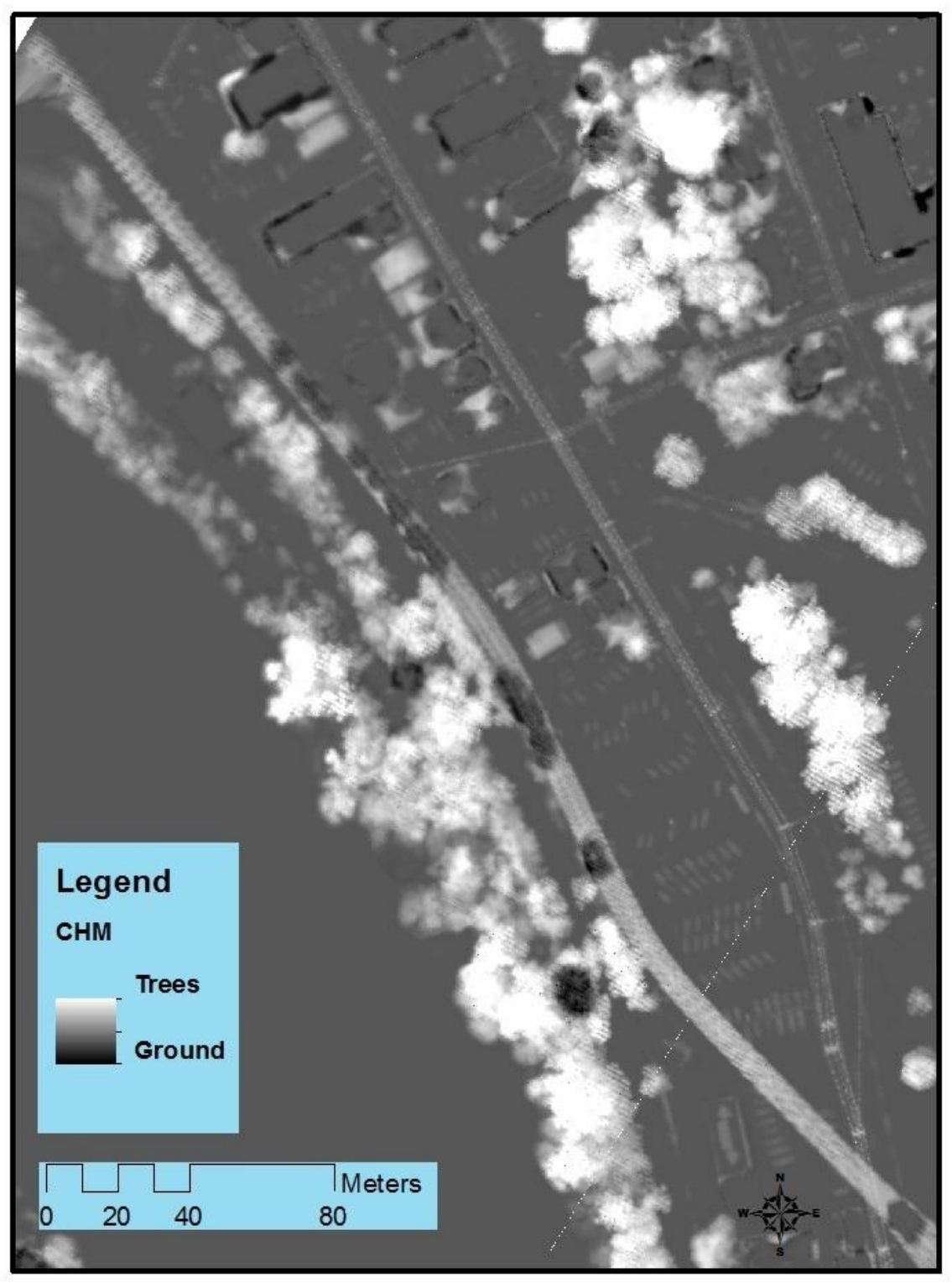

Figure 18: The CHM model on the banks of the Monongahela River. The trees along the west edge of the image are well represented, but the elevated track of the Personal Rapid Transit (PRT) which runs down the center of the image, has only portions which were removed, while much of it was retained in the final CHM. 


\section{$\underline{\text { Segmentation Methods }}$}

\section{Watershed Segmentation}

Although watershed segmentation tends to agglomerate deciduous trees where there are large clumps of trees with similar heights, watershed image segmentation is potentially more effective than TreeVaW in the situation where complex canopies have adequate troughs between adjacent tree crowns for the trees to be separated. It also gave a better representation of the structure of the vegetation in the study area since it conformed to the edges of the trees, at least within clumps of trees. Unfortunately, when tree crowns were close together but very similar in height, the watershed segmentation was less effective at separating individuals, and would often combine numerous trees into a single polygon.

Whereas TreeVaW works best with trees which are separated (Andersen 2009), watershed segmentation provided an adequate segmentation for the classification of other deciduous and coniferous trees, so it would be best for segmenting tree populations which are complex, such as in West Virginia, and in mixed forests of deciduous and coniferous species in both rural and urban environments.

\section{$\underline{\text { TreeVaW }}$}

The TreeVaW segmentation was effective at finding the apex of each tree, but it was less effective at predicting the size of the tree crowns. Many of the crown diameters were underestimated (Figure 19). TreeVaW also missed a few apparently distinct trees, perhaps because the level of smoothing was too extreme for the program to discern the tree as distinct from the 
ground. However, other studies using the TreeVaW software concluded that separating trees is difficult in close canopy tree stands (Andersen 2009). TreeVaW does not have the problem which watershed segmentation had of agglomeration of multiple canopies into a single polygon in areas of dense canopy cover. In areas where the forest is rather uniform without many gaps, TreeVaW may produce better results than watershed segmentation.

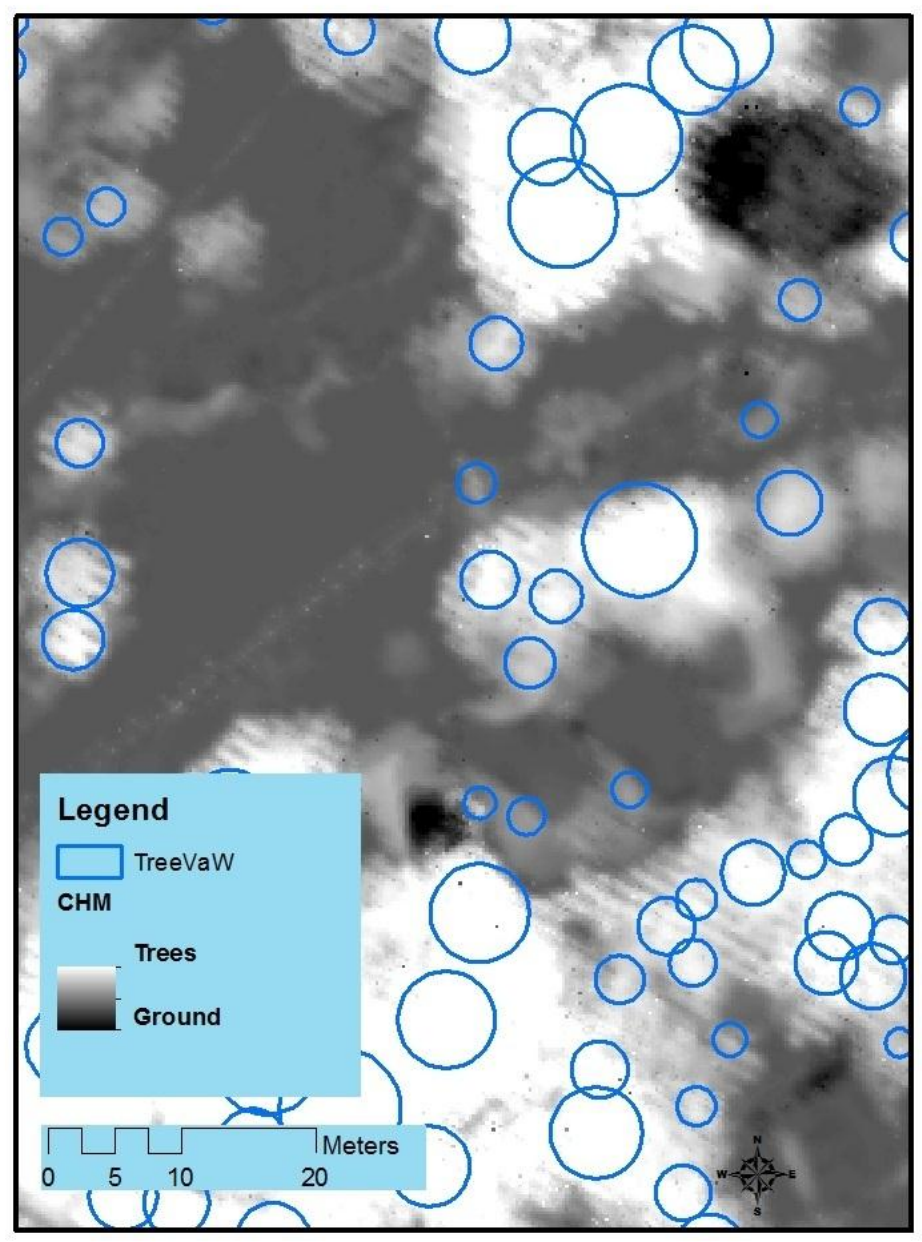

Figure 19: Under-representation of trees by TreeVaW segmentations (blue) compared with actual trees (white) 
Judging from the classification accuracy of the TreeVaW segmentation, when only adequate segmentations were considered, the TreeVaW did quite well, sometimes exceeding the results from the watershed segmentation (Tables 11 and 12). The accuracy of the TreeVaW segmentation in the Table 3 segmentation suggests a circle may be a beneficial way to segment a tree canopy which is complex, because it still retains enough of the internal attributes to make high quality identifications. It may also be useful in creating seeded watershed segmentations in the Idrisi Selva software, especially since it already produces points at tree apices. Using this approach, the watershed segmentation and the TreeVaW program could potentially be combined to form another segmentation method.

\section{$\underline{\text { Classification }}$}

The best combination of segmentation and classification methods for classifying Ailanthus trees, when the trees were correctly segmented, is the NN classification of the TreeVaW segmentation (Table 11). Judging from these results, further refinement of the TreeVaW segmentation may facilitate a highly accurate tree species classification when combined with high resolution data and powerful classifiers such as CART and NN.

The CART classification was very effective for classification in general. CART has the benefits of being simple to use, fast to process, and provides a consistent level of accuracy. CART did have difficulty with separating the Ailanthus altissima from other deciduous trees, at least compared to the SVM classification, which outperformed the CART classification when 
applied to both watershed and TreeVaW segmentations in the preliminary dataset and for overall accuracy using all the field data (Table 11).

The main benefit of NN is that it can create classification schemes based upon complex decision boundaries, as with tree species attributes. However, this makes it difficult to implement, as it has many inputs and the result can vary greatly with small changes in each parameter. In addition, since the NN classification has so many parameters, it is difficult to find the optimal combination for each dataset, and it is difficult to disprove the suggestion that another combination may have resulted in a higher accuracy.

The SVM classification was very effective at finding Ailanthus individuals, producing a high users' accuracy, although it seemed to misclassify more coniferous trees than CART. This may indicate that SVM is more strongly affected by the number of samples, since I have only 57 coniferous tree samples and 105 Ailanthus samples, and the SVM was very good with classifying the Ailanthus trees, and not as good as classifying the coniferous trees. The benefits of SVM are that it is easy to manage, having very few parameters, and that it is proficient at handling many attributes relatively quickly. Unfortunately, it is not easy to find out which attributes the SVM utilizes to classify the data. This makes it more difficult to improve upon the classification by getting more accurate data from the attributes which are most effective.

\section{Generalizations from the Supplemental Dataset}

The supplemental July 2008 dataset was included to allow a comparison with the results of my primary dataset, to see how the findings of this research could be generalized, and applied 
to other LiDAR datasets with a different point density. The results for the supplemental dataset were much less accurate than for the primary dataset. Identifying the reasons for this lower accuracy is important for making recommendations for future studies.

I used the exact same sequence of analysis on the supplemental dataset as I used for the primary datasets (with the small exception of adjusting some of the parameters such as the number of points used in the IDW, to fit the dataset).

The supplemental dataset shows some very important factors in the quality of the CHM which affect the segmentation and classification accuracy, including LiDAR penetration, LiDAR point spacing, and segmentation accuracy. 


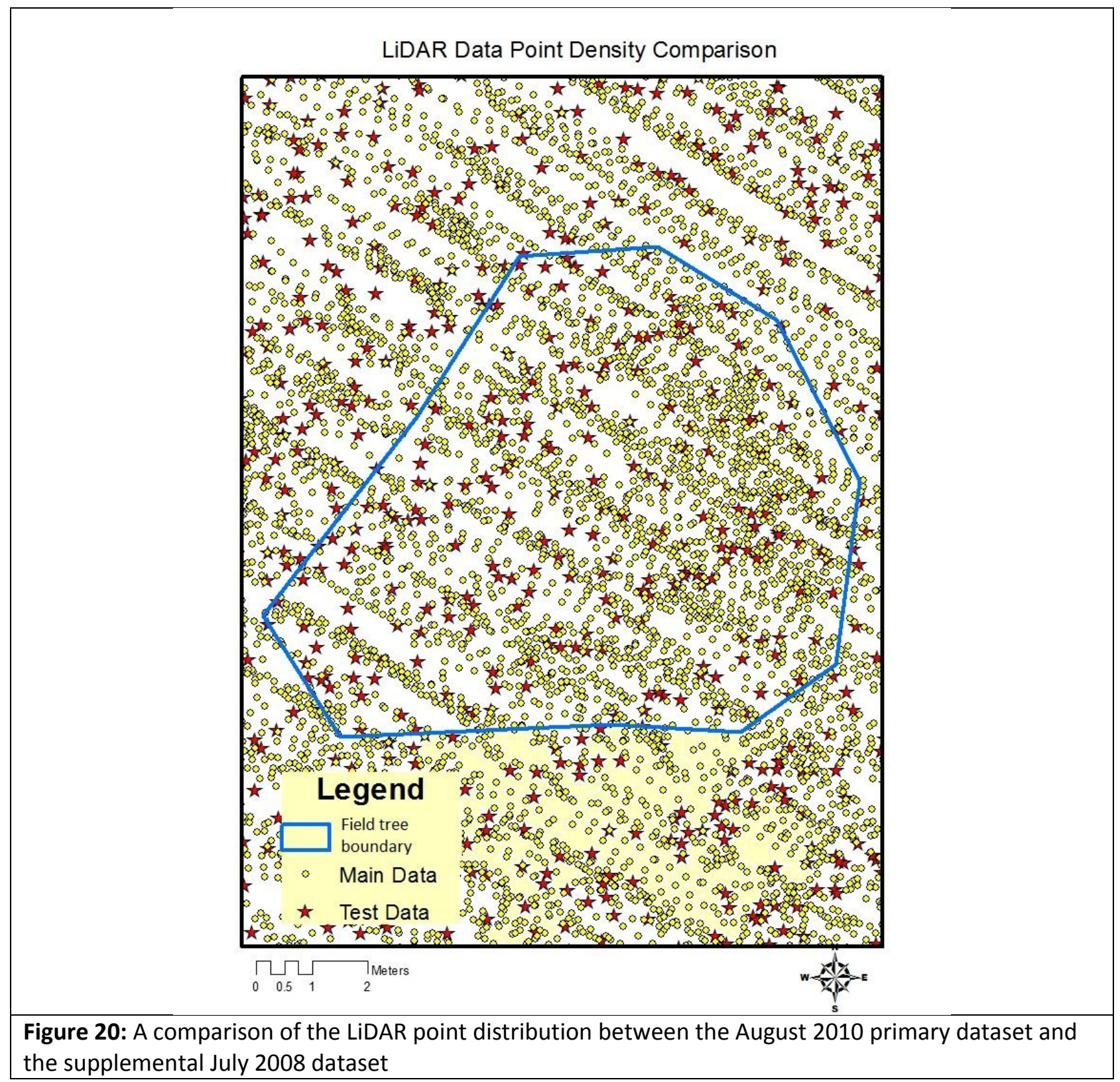

The number of points per $\mathrm{m}^{2}$ was greater in the primary dataset than the supplemental dataset (Figure 20), and this was likely the main cause in the less accurate classifications with the supplemental dataset. The test CHM did not produce well-defined trees, and considering the smaller number of points and the time of year it was taken, there was a problem with 
accurately penetrating the denser tree canopies (Figure 21). The trees with very thick canopies which were not adequately penetrated by the LiDAR beams were not included in the analysis because they could not be separated from the ground values since the last returns were likely from within the tree canopy and thus categorized as ground points. This difficulty resulted in less trees being included in the $\mathrm{CHM}$, and thus, the segmentation. This supplemental dataset shows clearly how this series of steps in determining tree species is heavily dependent upon the point density of the LiDAR data, the ability for the lasers to penetrate the tree crowns, and the quality of the CHM.

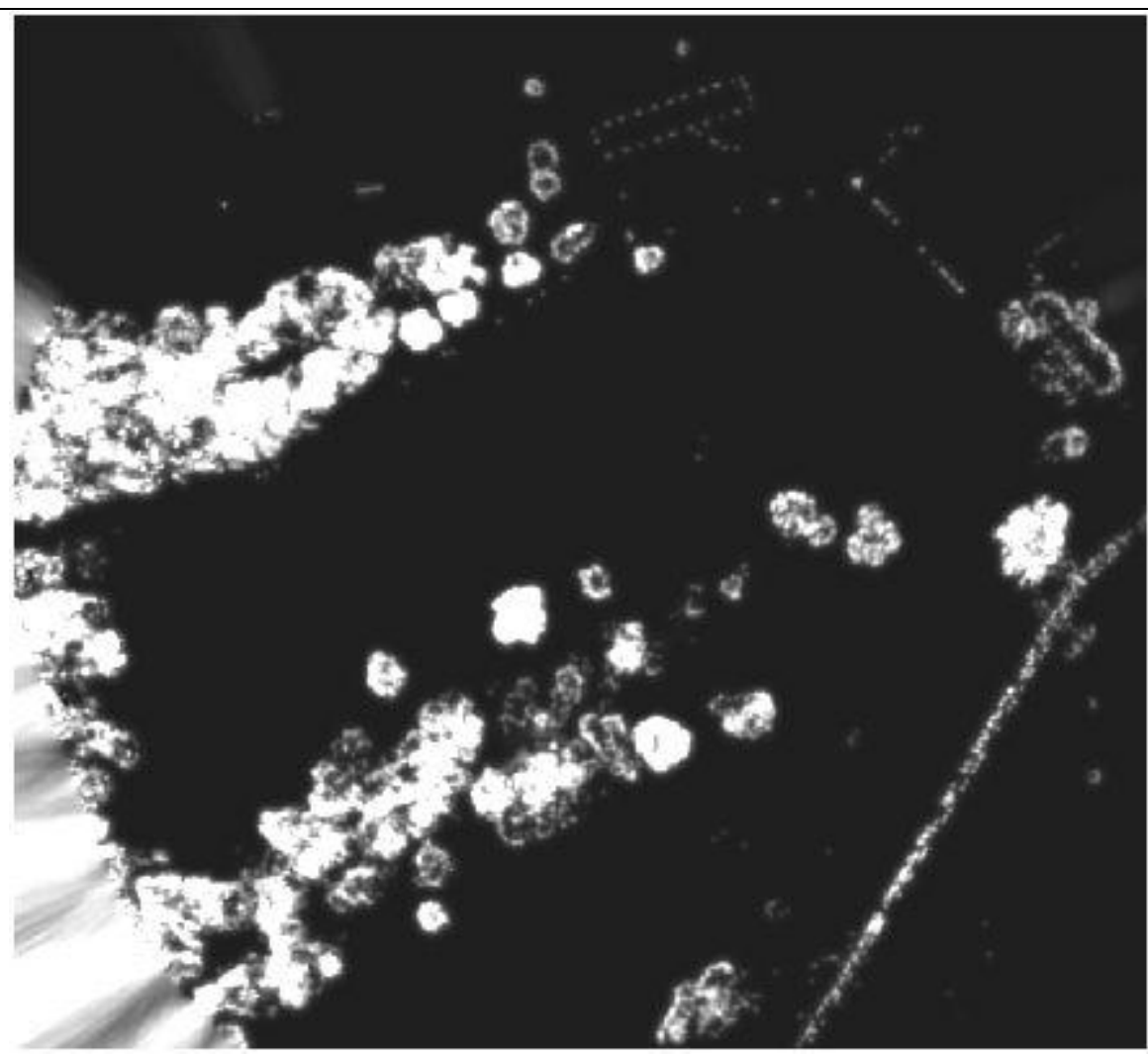

Figure21: CHM of the test data where trees (white) are not fully outlined since the LiDAR points did not penetrate the crown and the canopy filter was not able to differentiate canopy from ground. This resulted in crescent-like trees. 


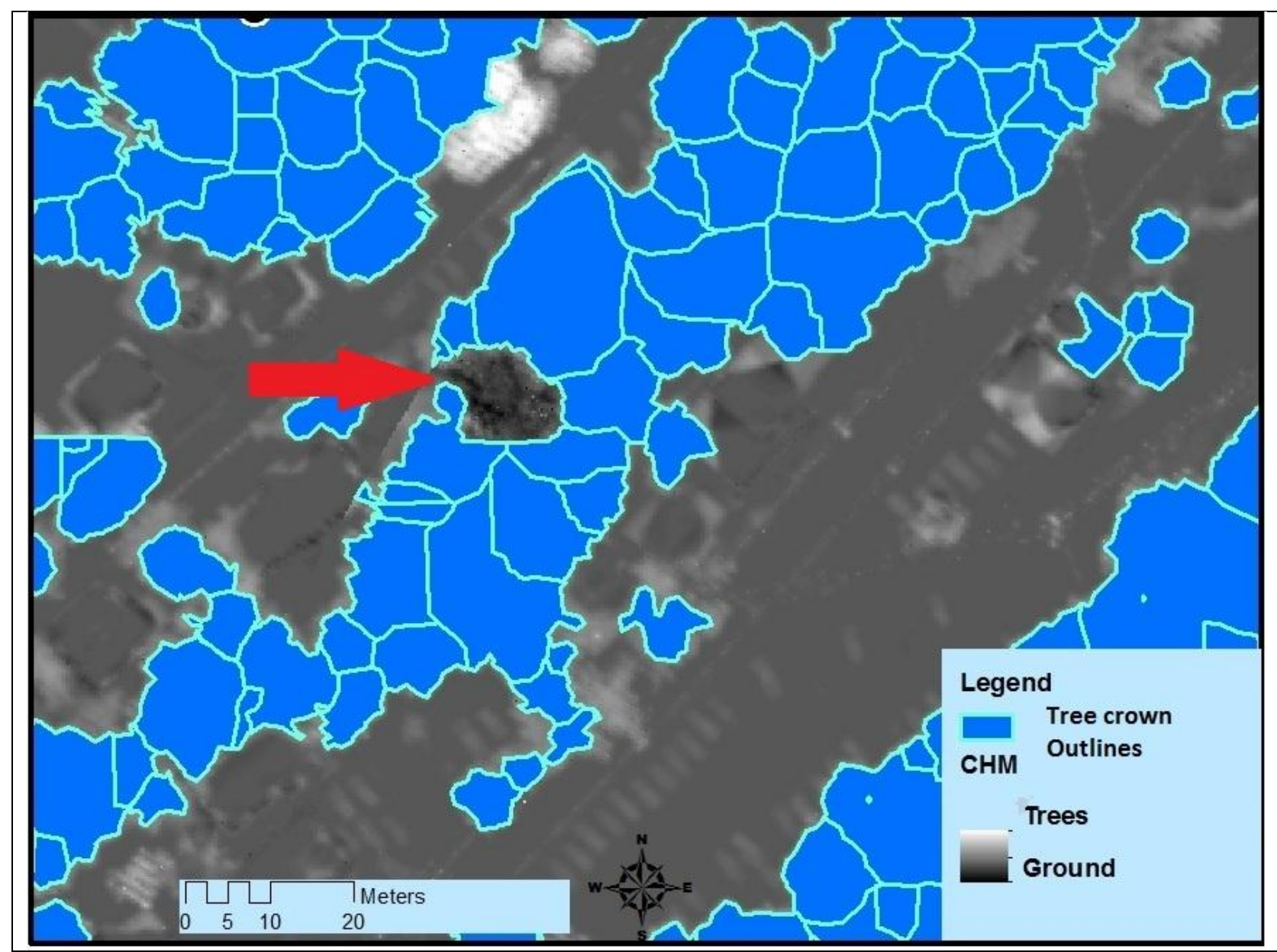

Figure 22: Trees which were not included in the segmentation because the crown was not penetrated by the laser beam. The arrow indicates a tree missing in the CHM.

LiDAR point penetration is needed to have a tree crown present in the $\mathrm{CHM}$. Without point penetration in the LiDAR data, no matter how well the canopy top is covered, it is eliminated from the $\mathrm{CHM}$ and appears as if no tree exists (Figure 22). This dramatically decreases the accuracy of the classification since the tree cannot be categorized correctly if it is not present in the $\mathrm{CHM}$ or the subsequent segmentation.

Interpolation choice is also factor, in creating a high quality CHM. IDW seemed to handle the supplemental July 2008 dataset $\left(4-5\right.$ points $/ \mathrm{m}^{2}$ ) less effectively than it handled the 
preliminary dataset (11-12 points $\left./ \mathrm{m}^{2}\right)$. IDW may be less effective with low point densities and tends to create "dimpled" trees which can be seen in the supplemental July 2008 dataset (Figure 23). Changing the IDW radius per points setting does lesson the dumpling effect in the trees, but also lessons the quality of the TreeVaW segmentation.

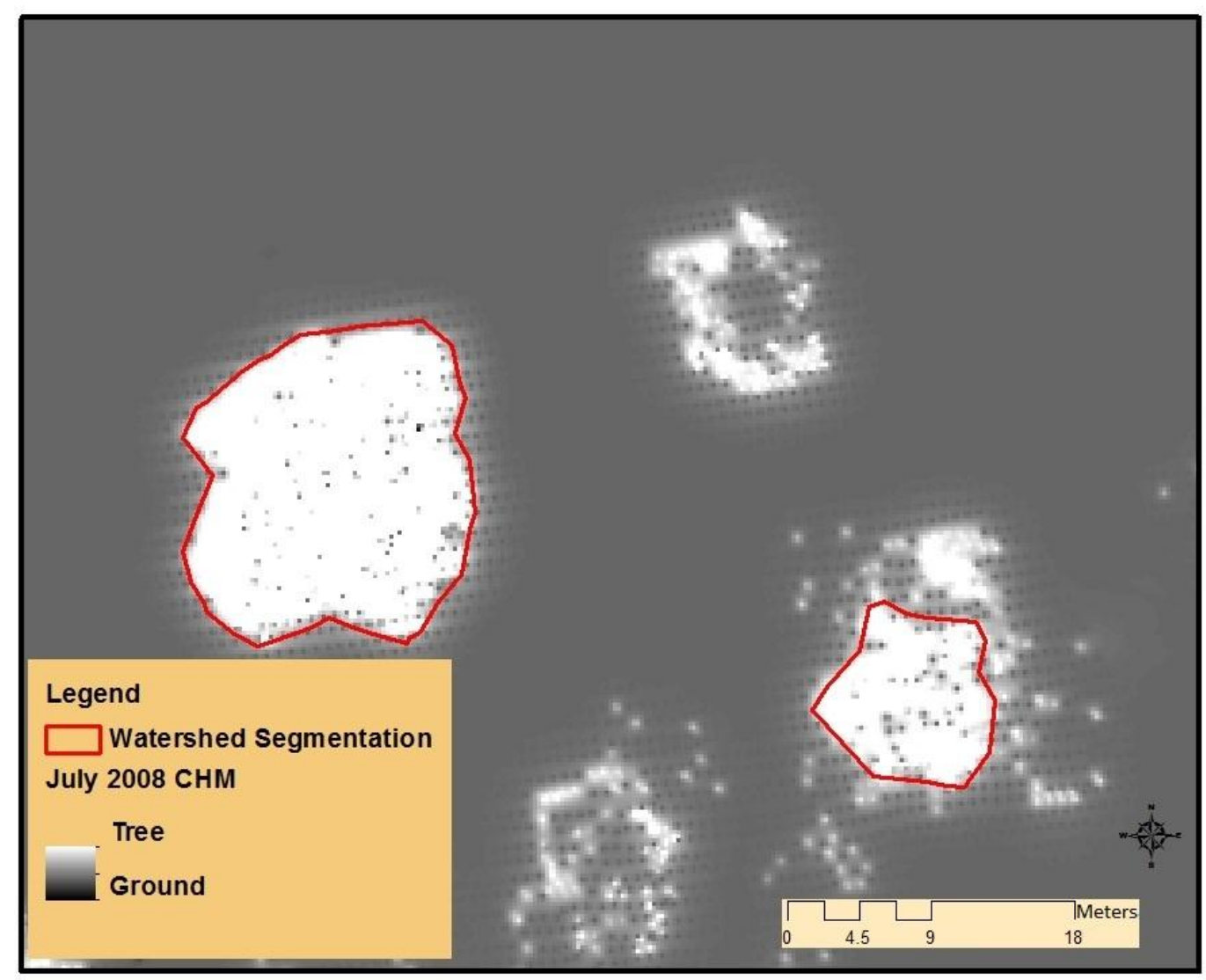

Figure 23: CHM with segmentation results superimposed using the supplemental July 2008 dataset. Interpolation artifacts which produces dimpled trees.

Segmentation errors include under-representation, over-segmentation or undersegmentation, each of which is ultimately caused by the quality of the CHM. 
Under-segmentation in TreeVaW segmentation occurs typically when the segments are too small, since it is using a regression to determine each crown height, and it is heavily dependent upon the quality of the CHM to produce accurate heights (Figure 24). Many of the tree crowns are not given a very accurate diameter value, causing large trees to have very small segments.
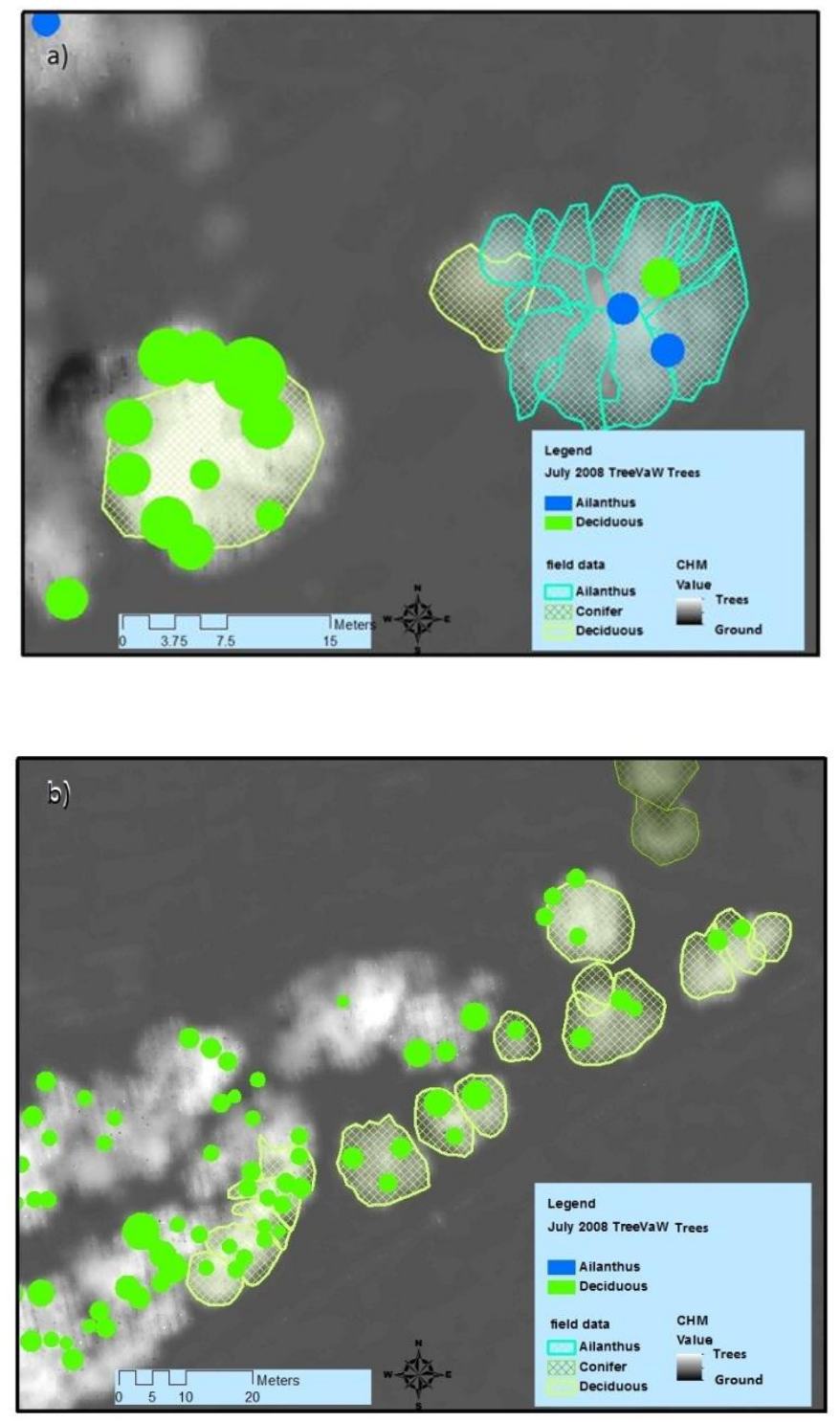

Figure 24: CHM with overlain classified TreeVaW segmentation and associated field data undersegmentation of the supplemental July 2008 dataset. (a)Zoomed in view. (b) A view covering a larger area. 


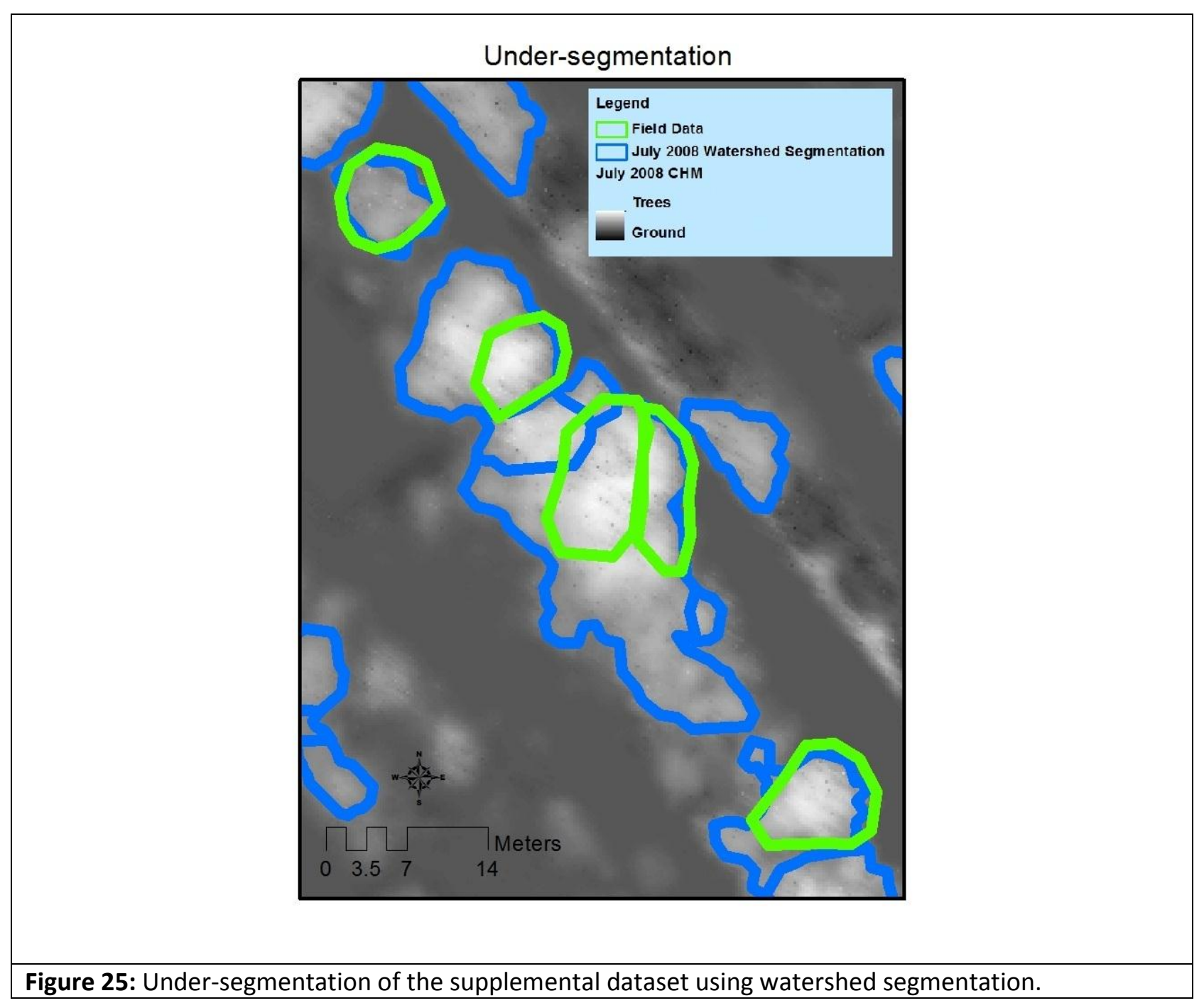

Over-segmentation occurs commonly more commonly with watershed segmentations

(Figure 25), especially in areas of dense deciduous crown canopy (Figure 26). 

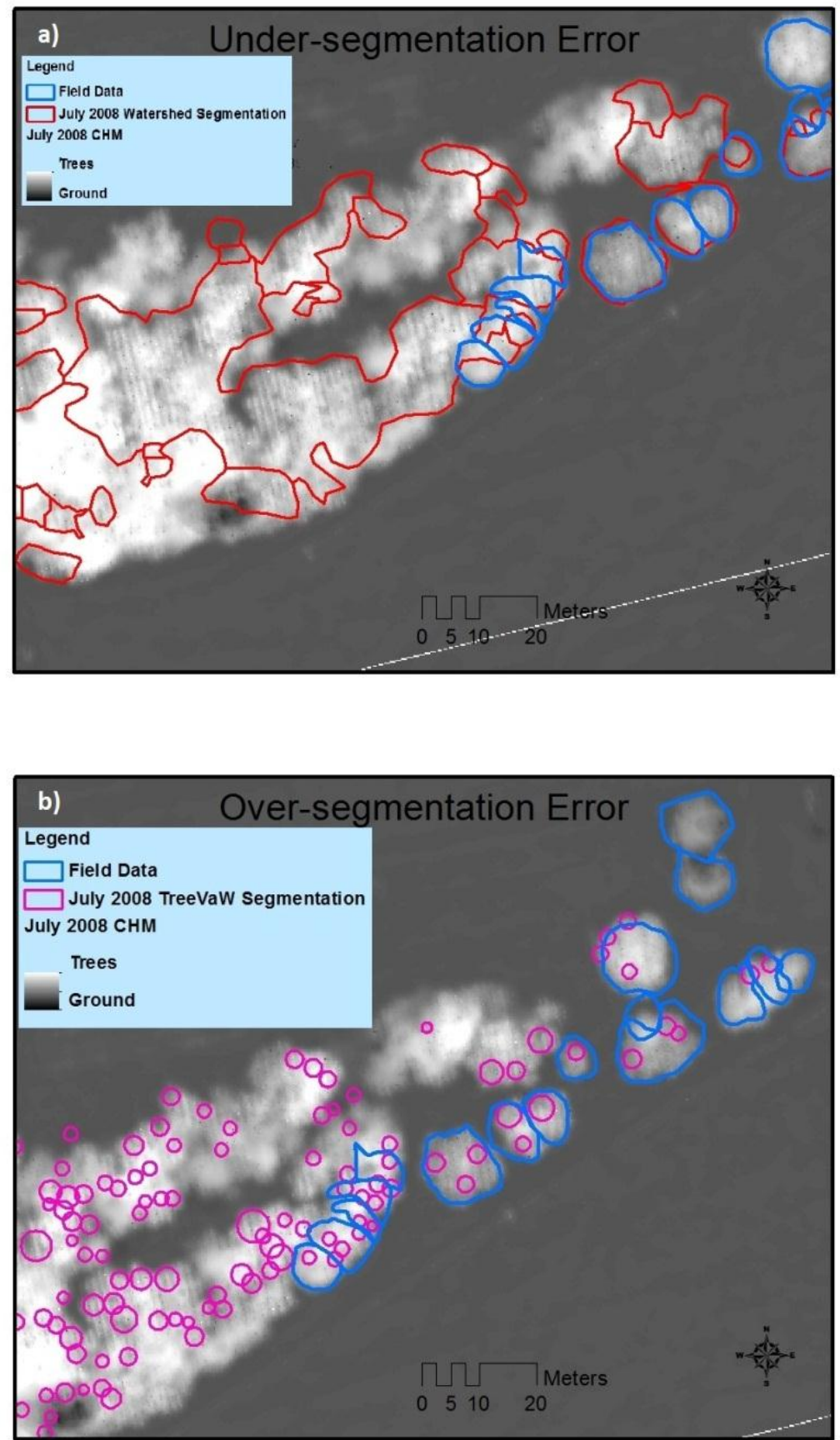

Figure 26: Over-segmentation and under-segmentation of the supplemental July 2008 dataset. (a) under-segmentation (b) over-segmentation 


\section{$\underline{\text { Limitations }}$}

The main limitation to high accuracy of the segmentations and classifications is the quality of the $\mathrm{CHM}$ because it affects the quality of the segmentation. Inaccurate tree segments make the classification of the data less representative of real-world measurements, and thus harder to classify an entire area.

The quality of the field data is also a limitation, and this has consequences both for the training of the classifiers, and for the evaluation of the results. Tracing tree crowns by walking around the trees on steep slopes with a GPS receiver was challenging, especially in areas with thick brush. GPS signals are attenuated by vegetation cover, and vegetation often limits the number of satellites visible to the unit. In addition, the short time windows where satellite positions were optimal also reduced signal quality.

The number of samples taken in the field data is also a limitation, since both test data and training data are derived from the field data. In order to have a good classification, a larger number of field data might improve the classification, since the sample might capture the range of tree properties more effective. 


\section{CHAPTER SIX}

\section{CONCLUSION}

The results of this research suggest that LiDAR data can be used to identify Ailanthus altissima species. The results are best when the LiDAR point density is high, and penetration of the tree crowns by the lasers is adequate. Other factors which affect the quality of the classification output are: the quality of the field data, the size of the field dataset, and the inadequacy in the classification and segmentation parameters. In order to create more accurate classifications, the important aspects are the quality of the $\mathrm{CHM}$, the subsequent segmentation, and the use of the most effective attributes tailored to the specific species of interest.

The most accurate segmentation is watershed segmentation. The most accurate classification for classifying Ailanthus, other deciduous and coniferous trees is CART. The most used attributes in the CART classification were the standard deviation of canopy slope, and percentages of points which were classified as ground, first return or intermediate returns for each tree.

In future research, a number of methodological improvements could increase Ailanthus classification accuracy. Differentiation of male and female Ailanthus in the classification would be useful since female Ailanthus trees, which have seed clusters present all year long, did cause a slightly different laser intensity value. Additional exploitation of the intensity values of the LiDAR points within the tree canopy may also increase classification accuracy, and this could potentially be done if it were possible to normalize second and subsequent returns to the intensity of the first returns. Perhaps by the inclusion of using landscape context, such as the 
position of the tree relative to its closest neighbors, could increase accuracy since a tree standing alone in a field has a different profile than a tree which is closely surrounded on all sides, or is on the edge of a forest. For a landscape context to be included, a larger, more extensive field dataset would need to be collected. Lastly, the TreeVaW segmentation could be more properly modified to accommodate the clustering of Ailanthus trees and thereby increase the accuracy of segmentation, and thus, classification.

The main problem I encountered with this analysis is that most common software packages were not written to deal with the vast quantity of data associated with LiDAR datasets. Most software systems were originally created to handle a small number of single points, and they have not developed a strategy for dealing with the LiDAR point cloud. The LiDAR point cloud is much larger than the typical single-point dataset, and tends to overload the software quickly. Even a problem as basic as file format is an issue; not all read the LiDAR standard file format (.Las) (Graham, 2005), and even fewer can directly work with the .Las format. This makes it difficult to use large LiDAR datasets, especially if multiple software packages are needed because data conversion has to be done several times as the data is passed between software systems.

Regardless of the difficulty of dealing with LiDAR data, the process that I have outlined does show promise in species identification. 


\section{REFERENCES}

Andersen, H. 2009. Using airborne light detection and ranging (LIDAR) to characterize forest stand condition on the Kenai peninsula of Alaska. Western Journal of Applied Forestry. 24, 95-102.

Asner , G., Knapp D., Boardman J., Hughes R., Jones M., Kennedy-Bowdoin D., Martin R. 2008. Invasive species detection in Hawaiian rainforests using airborne imaging spectroscopy and LiDAR. Remote Sensing of Environment. 112: 1942-1955.

Bischof, H., Schneider, W., and Pinz, A., 1992. Multispectral classification of landsat-Images using neural networks. IEEE Transactions on Geosciences and Remote Sensing, 30(3): 482-490.

Bodley, C., and Friedl, M. 1997. Decision tree classification of land cover from remotely sensed data. Remote Sensing of the Environment, 61: 399-409.

Borgefors, G. (1986). Distance transformation in digital images. Computer Vision, Graphics, and Image Processing, 34(3), 344-371.

Bork, E. W., and Su, J. G. 2007. Integrating LIDAR data and multispectral imagery for enhanced classification of rangeland vegetation: A meta analysis. Remote Sensing of Environment, 111: 11-24.

Brandtberg., T. 2007, Classifying individual tree species under leaf-off and leaf-on conditions using airborne lidar. ISPRS Journal of Photogrammetry and Remote Sensing, 61: 325-340

Brandtberg, T. and Warner., T. 2006. High resolution remote sensing. In: G. Shao and K. M. Reynolds (eds.), Computer Applications in Sustainable Forest Management, Springer Verlag, Dordrecht, Netherlands, Chapter 2, pp. 19-41.

Brandtberg, T., Warner, R. Landenberger, and J. McGraw, 2003, Detection and analysis of individual leaf off tree crowns in small footprint, high sampling density LIDAR data from the eastern deciduous forest in North America. Remote Sensing of Environment, 85, pp.290-303.

Canham, C., and Knapp, L. 2000. Invasion of an old-growth forest in New York by Ailanthus altissima: sapling growth and recruitment in canopy gaps, Journal of the Torrey Botanical Society, 127(4): 307-315.

Chen, Q., Gong, P., Baldocchi, D., and Kelly, M. 2006. Isolating individual trees in a savanna woodland using small footprint lidar data. Photogrammetric Engineering \& Remote Sensing, 72 (8): 923-932.

Congalton, R. 1991. A review of assessing the accuracy of classification of remotely sensed data. Remote Sensing of Environment, 37(1): 35-46

Craine, J. 2009. Resources Strategies of Wild Plants. Princeton University Press. Princeton New Jersey.

Czaplewski, R., and Stehman, S. 1998, Introduction to special issue on map accuracy. Remote Sensing of Environment. 64: 331-344. 
De'ath, G., and Fabricius, K. 2000. Classification and regression trees: A powerful yet simple technique for ecological data analysis. Ecology, 81(11): 3178-3192.

Donoghue, D., Watt, P., Cox, N., and Wilson, J. 2007. Remote sensing of species mixtures in conifer plantations using LiDAR height and intensity data. Remote Sensing of Environment. 110:1. 509-522.

Dussault, C., Ouellet, J., Courtois, R., and Huot, J. 2001. Influence of satellite geometry and differential correction on GPS location accuracy. Wildlife Society Bulletin. 29(1): 171-179

Falkowski, M., Martinuzzi, S., Evans, J., Gessler, P., Hudak, A. 2009, Characterizing forest succession with lidar data: An evaluation for the Inland Northwest, USA. Remote Sensing of Environment, 113: 946-956.

Graham, L. 2005, The LAS 1.1 Standard. Photogrammetric Engineering and Remote Sensing, 71(7), 777780.

Ham, J., Chen, Y., Crawford, M., and Ghosh, J. 2005. Investigation of the random forest framework for classification of hyperspectral data. IEEE Transactions on Geoscience and Remote Sensing. 43(3): 492501.

Heikkinen, V., Tokola, T., Parkkinen, J., Korpela, I., and Jaaskelainen, T., 2010. Simulated multispectral imagery for tree species classification using support vector machines. IEEE Transactions on Geoscience and Remote Sensing, 48(3): 1355-1364.

Holmgren, J., Persson, A., Soderman, U. 2008. Species identification of individual trees by combining high resolution LiDAR data with multi-spectral images. International Journal of Remote Sensing. 29(5): 1537-1552.

IDL Online Help. 2011. Creating Image Object Boundaries. Retrieved from http://star.pst.qub.ac.uk/idl/Creating Image Object Boundaries.html Accessed 4 April 2012

Kowarik, I. 1995. Clonal growth in Ailanthus altissima on a natural site in West Virginia. Journal of Vegetation Science, 6: 853-856.

Landenberger, R., and Kota, N. 2006. Seed dispersal of the non-native invasive tree Ailanthus altissima into contrasting environments. Plant Ecology. 9: 197-211.

Lawrence, R., and Wright, A. 2001. Rule-based classification systems using classification and regression tree (CART) analysis. Photogrammetric Engineering and Remote Sensing, 67(10): 1137-1142.

Leigh, C., Kidner, D., and Thomas, M. 2009.The use of LiDAR in digital surface modeling: Issues and Errors. Transactions in GIS, 13(4): 345-361

Mangan, A., and Whitaker, R. 1999. Partitioning 3D surface meshes using watershed segmentation. IEEE Transactions on Visualization and Computer Graphics, 5(4): 308-321. 
Martin, M., Newman, S., Aber, J., Congalton, R. 1998. Determining forest species composition using high spectral resolution remote sensing data. Remote Sensing of Environment. 65: 249-254

Melgani, F., and Bruzzone, L. 2004. Classification of Hyperspectral Remote Sensing Images with Support Vector Machines. IEEE Transactions on Geoscience and Remote Sensing, 42(8): 1778-1790.

Mitasova, H., Mitas, L., and Harmon, R. 2005. Simultaneous spline approximation and topographic analysis for lidar elevation data in open source GIS. IEEE Geosciences and Remote Sensing letters. 2(4) pp. 375-379.

Morsdorf, F., Nichol, C., Malthus, T., and Woodhouse, I. 2009. Assessing forest structural and physiological information content of multi-spectral LiDAR waveforms by radiative transfer modeling. Remote Sensing of Environment, 113: 2152-2163.

Myers, D. E. 1994: Spatial interpolation: an overview. Geoderma 62, 17-28.

Orka, H., Naesset, E., Bollandsas, O. 2008, Classifying species of individual trees by intensity and structure features derived from airborne laser scanner data. Remote Sensing of Environment. 113 (6) 1163-1174.

Palleja,T., Tresancheza, M., Teixidoa, M., Sanz, R., Palacina, J., and Rosellb, J. 2010. Sensitivity of tree volume measurement to trajectory errors from a terrestrial LIDAR scanner: Agriculture and Forest Meteorology, 150: 1420-1427.

Popescu, S., Wynne, R., and Nelson, R. 2002. Estimating plot-level tree heights with lidar: local filtering with a canopy-height based variable window size. Computers and Electronics in Agriculture, 37: 71-95.

Popescu, S., Wynne, R., and Nelson, R. 2003. Measuring individual tree crown diameter with lidar and assessing its influence on estimating forest volume and biomass. Canadian Journal of Remote Sensing, 29(5): 564-577.

Popescu, S., Wynne, R., and Zhao, K. 2009. Lidar remote sensing of forest biomass: A scale-invariant estimation approach using airborne lasers. Remote Sensing of Environment, 113: 182-196.

Queen, L., Seielstad, C., Suratno, A. 2009, National Center for Landscape Fire Analysis, pp. 1-10, Retrieved on 2-25-2011 from http://firecenter.umt.edu/files/publications/

Reitberger, J., Krzystek, P., and Stilla, U. 2006. Analysis of full waveform LiDAR Data for tree species classification. Remote Sensing and Spatial Information Sciences, 36 (Part 3): 228-233.

Shan, J., and Toth, C. 2008. Topographic Laser Ranging and Scanning: Principles and Processing, CRC Press, New York. 
Song, J., Han, S., Kim, Y., and Yu, K. 2002. Assessing the possibility of land-cover classification using lidar intensity data. International Archives of Photogrammetry, Remote Sensing and Spatial Information Sciences, 34 (Part 3B): 259-262. 


\section{APPENDICES}

\section{Appendix 1: Watershed Code:}

The following Code is written in IDL, for use in the ENVI software. The majority of the code was obtained from IDL Online Help (2001):

; \$ld://depot/idl/IDL_70/idldir/examples/doc/image/watershedexample.pro\#1 \$

; Copyright (c) 2005-2007, ITT Visual Information Solutions. All rights reserved.

; Edited by Cassidy Rhea

PRO WatershedExampleEdited

; Prepare the display device.

DEVICE, DECOMPOSED = 0, RETAIN = 2

LOADCT, 0

; Select a CHM in the form of a JPG (First convert the raster data to Bitmap, then to JPG)

file = FILEPATH('CHM.jpg', ROOT_DIR=['G:'], \$

SUBDIR=['Thesis','CHM','point25m','sections','ENVI'])

; Convert the image to greyscale

READ_JPEG, file, img, /GRAYSCALE

;Invert the image, turning the 'trees' into 'valleys'

bimg = MAX(img) - img

; Get the image size, create a window and display the image in the window.

dims = SIZE(img, /DIMENSIONS)

WINDOW, 0, XSIZE = 3*dims[0], YSIZE $=2 * \operatorname{dims}[1], \$$

TITLE = 'Watershed segmentation of Trees'

; Display the original image.

TVSCL, bimg, 0

XYOUTS, 50, 444, 'Original Image', Alignment $=.5, \$$

/DEVICE, COLOR = 255

; Smooth the image and then display it alongside the original image.

smoothlmg = SMOOTH(bimg, 7, /EDGE_TRUNCATE)

TVSCL, smoothlmg, 1

XYOUTS, $(60+\operatorname{dims}[0]), 444$, 'Smoothed Image', \$

ALIGNMENT $=.5$, /DEVICE, COLOR $=255$ 
; Define the radius and create the structuring element, smaller radius creates more condensed ;watersheds.

radius $=35$

strucElem = SHIFT(DIST $(2 *$ radius +1$), \$$

radius, radius) LE radius

; Use the top-hat operator before using watershed to highlight bright areas within the image. tophat Img = MORPH_TOPHAT(smoothImg, strucElem)

; Display the top-hat image.

TVSCL, tophatImg, 2

XYOUTS, $(60+2 *$ dims[0]), 444, 'Top-hat Image', \$

ALIGNMENT $=.5$, /DEVICE, COLOR $=255$

; Determine the intensity value using a histogram as aguide. Stretch the image.

WINDOW, 2, XSIZE $=400$, YSIZE $=300$

PLOT, HISTOGRAM(smoothImg)

tophatImg = tophatImg $<70$

; Display the stretched image.

WSET, 0

TVSCL, tophatImg

XYOUTS, 75, 210, 'Stretched Top-hat Image', \$

ALIGNMENT $=.5, /$ DEVICE, COLOR $=255$

; Use the WATERSHED operator to create boundaries for the trees and display the results.

watershedImg = WATERSHED(tophatImg, CONNECTIVITY = 8)

TVSCL, watershedImg, 4

XYOUTS, (70 + dims[0]), 210, 'Watershed Image', \$

ALIGNMENT $=.5$, /DEVICE, COLOR $=255$

; Overlay the boundaries defined by watershed onto the original image.

img [WHERE (watershedlmg EQ 0)] = 0

TVSCL, img, 5

XYOUTS, (70+2*dims[0]), 210, 'Watershed Overlay', \$

ALIGNMENT $=.5, /$ DEVICE, COLOR $=255$

;Write Image to a file as bitmap, .jpg, or .gif

WRITE_IMAGE, 'watershed_out10.bmp', 'BMP', img

END 


\section{Appendix 2: Skewness Calculation:}

Skewness of height is not a feature currently offered in ArcGIS10, so I had to develop my own method for calculating skewness (Figure 27).

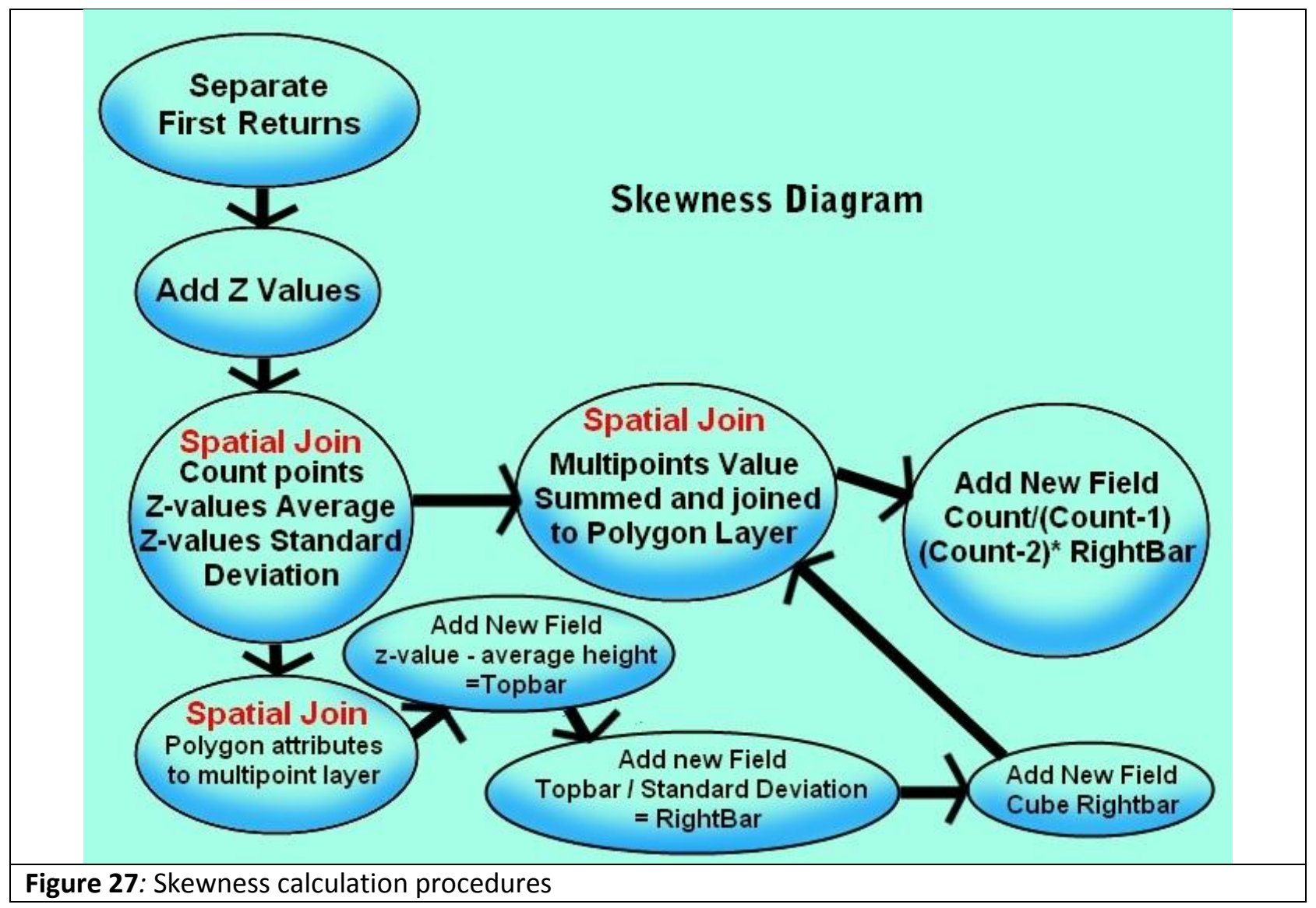




\section{Appendix 3: Fuzzy Overlap Calculation:}

The fuzzy overlap measure was designed by Brandtberg et al., and implemented by Dr. Timothy Warner and I. Figure 28 shows a schematic for the model in ERDAS Imagine 2011 software. Table 14 gives the associated formulas.

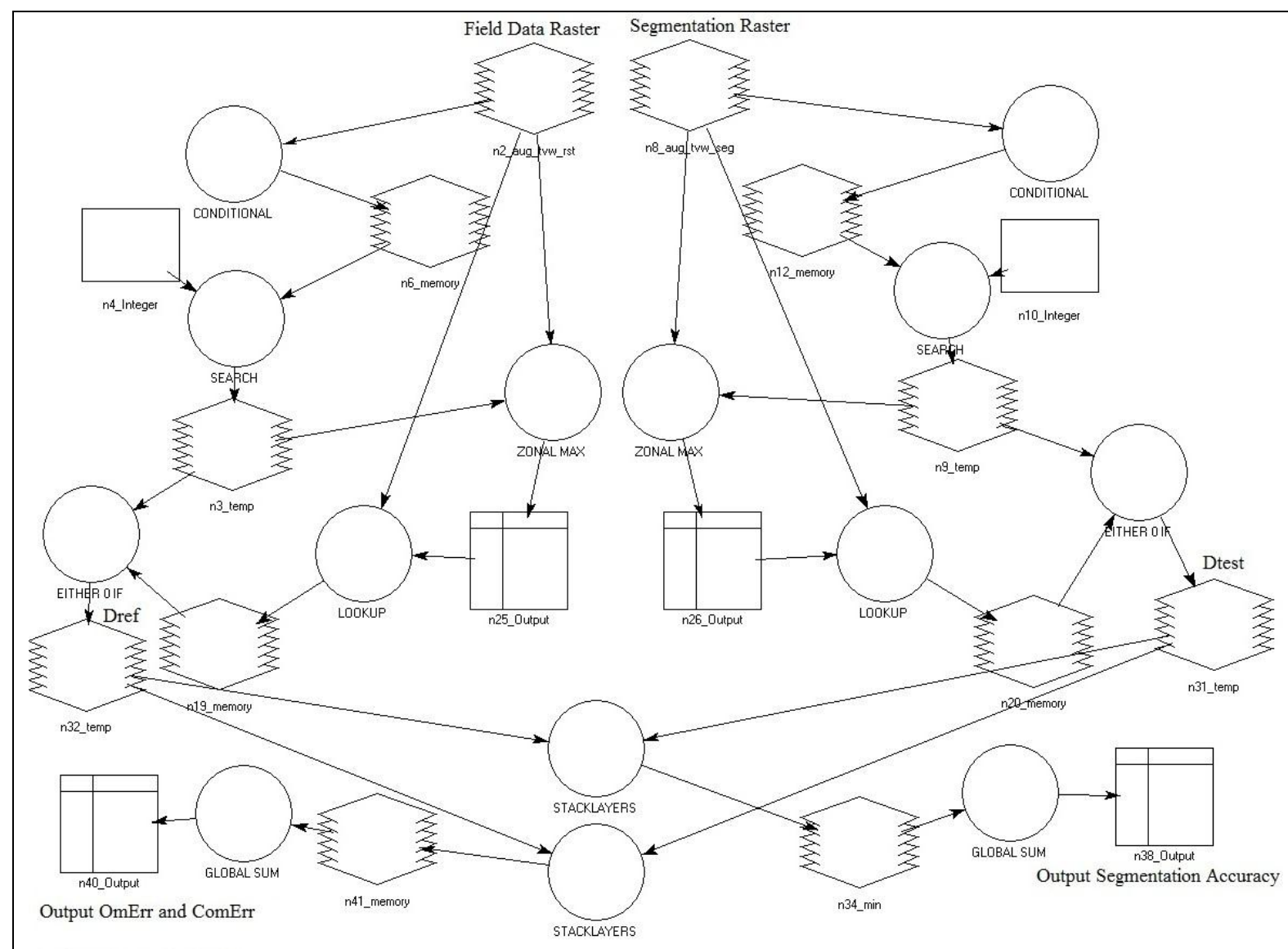

Figure 28: Fuzzy segmentation accuracy assessment model in Imagine 


\begin{tabular}{|c|c|}
\hline \multicolumn{2}{|c|}{ Table 15: The formulas for the operations in the left side of the model in Figure 24.} \\
\hline Section & Formula \\
\hline Conditional & CONDITIONAL $\{(\$$ n2_aug_tvw_rst $>0) 0$, (1) 1$\}$ \\
\hline Search & SEARCH ( \$n6_memory, \$n4_Integer, 1) \\
\hline Either if & $\begin{array}{l}\text { EITHER O IF ( \$n19_memory == 0) OR } \\
\text { \$n3_temp / \$n19_memory } \\
\text { OTHERWISE }\end{array}$ \\
\hline Stacklayers 1 & $\begin{array}{l}\text { STACKLAYERS }( \\
\text { MAX (\$n32_temp, \$n31_temp ), } \\
\text { MIN (\$n32_temp, \$n31_temp }) \\
\text { ) }\end{array}$ \\
\hline Stacklayers 2 & $\begin{array}{l}\text { STACKLAYERS }( \\
\text { CONDITIONAL }\left\{\left(\$ n 31 \_t e m p==0\right) \$ n 32 \_t e m p,(1)\right. \\
0\}, \\
\text { CONDITIONAL }\left\{\left(\$ n 32 \_t e m p==0\right) \$ n 31 \_t e m p,(1)\right. \\
0\} \\
1\end{array}$ \\
\hline
\end{tabular}

